
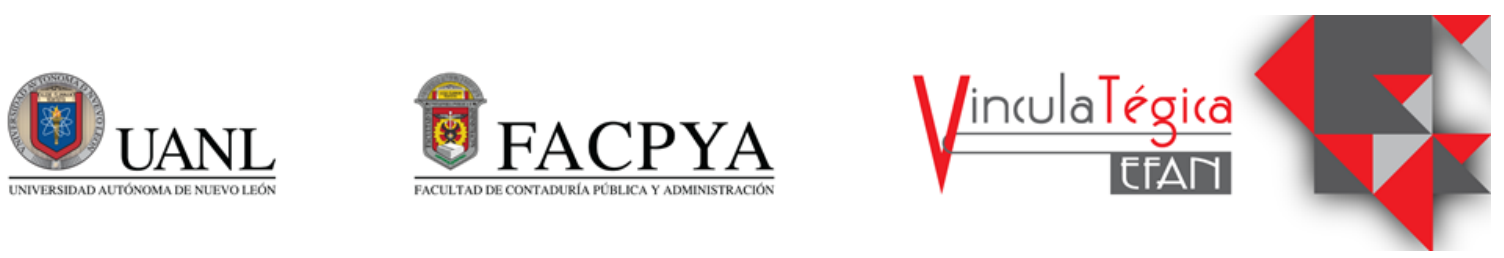

\title{
Factores que determinan la vinculación entre las empresas y las universidades que cuentan con Programa Dual de formación en Latinoamérica
}

\author{
Pedro Ariza Ricaldi ${ }^{1}$, Andrea Silva Niño ${ }^{2}$ y Caroline Mendoza Leclere ${ }^{3}$ \\ 1, Universidad de San Martín de Porres, Lima, Perú, parizar@usmp.pe; Lima, Perú. 51997677016 \\ 2. Universidad Autónoma de Bucaramanga, asilva4@unab.edu.co; Bucaramanga, Colombia. 3186867162 \\ ${ }^{3}$ Universidad. Interamericana para el Desarrollo; crmendoza@ unid.mx; México.525532247040 \\ Información del artículo revisado por pares \\ Fecha de aceptación: junio-2021 \\ Fecha de publicación en línea: diciembre-2021 \\ DOI: https://doi.org/10.29105/vtga7.2-46
}

\section{Resumen}

Este artículo examina individualmente los factores internos y los factores externos que las empresas valoran para vincularse con las universidades que cuentan con Programa Dual de formación en Latinoamérica. Se ha realizado un estudio exhaustivo y sistemático de la bibliografía existente, se han recopilado fuentes de información primaria y secundaria con literatura relevante. Finalmente desarrollamos un modelo de ecuaciones estructurales que puede proporcionar una comprensión más amplia de los factores que determina la relación en la vinculación de las empresas con las universidades con programa dual de formación, así como la influencia de variables mediadoras como los costos de formación y la participación empresarial en la vida académica y su incentivo para la vinculación empresa universidad. Los factores hallados que muestran una mayor vinculación son los factores internos caracterizados por los recursos humanos que las universidades proveen a la empresa para la transferencia de conocimiento y el impacto

económico generado por los proyectos ejecutados entre la empresa y la universidad. También se evidencia una relación positiva que favorece la vinculación cuando la empresa reconoce su participación en los quehaceres académicos. En ésta investigación proponemos un modelo sistematizado que permita a las universidades que buscan vincularse a las empresas potenciar sus factores internos los cuales son determinantes al momento de establecer una colaboración empresa y universidad.

Palabras clave: Recurso humano, impacto económico, vinculación empresa universidad, Programa Dual, ecuaciones estructurales.

\section{Abstract}


This article examines individually the internal factors and the external factors that companies value to link with the universities that have a Dual Training Program in Latin America. An exhaustive and systematic study of the existing bibliography has been carried out, primary and secondary information sources have been compiled with relevant literature. Finally, we develop a model of structural equations that can provide a broader understanding of the factors that determine the relationship between companies and universities with a dual training program, as well as the influence of mediating variables such as training costs and training costs. Business participation in academic life and its incentive for linking Company University. The factors found that show a greater link are the internal factors characterized by the human resources that the universities provide to the company for the transfer of knowledge and the economic impact generated by the projects executed between the company and the university. A positive relationship that favors bonding is also evidenced when the company recognizes its participation in academic tasks. In this research we propose a systematized model that allows universities that seek to link with companies to enhance their internal factors, which are decisive when establishing company-university collaboration.

Keywords: Human resource, economic impact, company - university link, Dual Program, structural equations.

\section{Introducción}

En muchos países la brecha entre lo aprendido en las escuelas y lo mínimo necesario para ser parte de las universidades sigue siendo crítico, esta brecha limita las posibilidades de las personas para lograr alcanzar una profesión y por tanto implica que las empresas dejan de contar con mano de obra calificada que les permita mantener y mejorar sus operaciones frente a una realidad altamente cambiante. El impacto de estas brechas se siente en la economía y se representa en el freno del desarrollo de los países DHLA (2018).

Como respuesta a este escenario, en Europa específicamente en Alemania, se ha desarrollado el Programa Dual, que logra unir los esfuerzos de la academia representada por las universidades, el Estado y los diversos actores sociales, en los que se encuentran las empresas, ésta unión de esfuerzos tiene como resultado mejorar constantemente los procesos de formación, cerrando brechas de conocimientos y alinear las necesidades reales de las empresas al proceso académico. De esta manera se busca brindar respuesta a una situación crítica detectada en la educación y contribuir en la mejora de la economía del país reduciendo la tasa de desempleo juvenil y logrando contar con personal disponible para las empresas mejor preparados.

La relación empresa universidad se ve reflejada en el exitoso Programa Dual, el éxito en Alemania radica en ser un país altamente innovador, la especialización de sus empresas $(90 \%$ de las Pymes del total de empresas) han identificado nichos de mercado donde se desarrollan y evolucionan constantemente siendo altamente competitivas y sumado a la buena gestión se encuentra el Programas Dual, donde las universidades participan en la formación del recurso humanos, esta relación logra la creación de currículo de estudio acorde a las necesidades empresariales, Programas como la Duale Hochschule Baden-Württemberg, han servido como guía para demostrar que esta relación logra un impacto alto en las empresas, impacto económico, sostenibilidad, recurso humanos especializado y mejora permanente de sus procesos entre otros. 
Alemania cuenta con la menor tasa de desempleados jóvenes de Europa junto a Austria y Suiza que también cuentan con el Programa Dual (tasa de desempleo al 2017, $6 \%$ ), mientras que otros países mediterráneos que no cuentan con Programa Dual han alcanzan el $40 \%$ de desempleo juvenil. DHLA (2018). OIT (2018).

Uno de los pilares del Programa Dual es la relación que las empresas puedan gestar con las universidades. La investigación acerca de esta relación ha impulsado la búsqueda de teorías, percepciones y nuevas orientaciones que puedan brindar un mejor alcance y entendimiento. Estos esfuerzos están mejor representados por el análisis empírico realizado por escritores como Alvarado-Borrego, (2000), Siqueira et al, (2009), López, Martínez, Medellín, Scanlon, \& Solleiro, (1994), Etzkowitz \& Leydesdorff, (2000). Laursen \& Salter, (2004), Vega \& Jurado et al, (2008). Sin embargo el trabajo de estos investigadores brinda la oportunidad de responder a dos limitantes importantes.

Primero, el problema es metodológico. Aunque la mayoría de estos estudios afirman explicar las razones de la vinculación empresa universidad, las propuestas son empíricas, con excepción del estudio de DHLA, (2018) el cual cuenta con literatura y resultados de corte cuantitativas y cualitativa, este último podría ser complementado con los estudios de las empresas que participan en el Programa Dual.

Segundo problema. Los estudios proponen la participación de la universidad en la empresa desde un enfoque de la universidad como "consultor o que brinda un servicio" de investigación y desarrollo. Probablemente si el enfoque es donde la universidad acompaña a la empresa con participación de su producto principal "estudiantes universitarios" permita conocer mejor la razón de vincularse por parte de la empresa con la universidad.

Todavía está pendiente una síntesis satisfactoria de la variada evidencia empírica aplicada a un Programa Universitario.

Lo que este artículo propone hacer es proporcionar un modelo que explique los factores internos y externos que determinan la vinculación de la empresa a la universidad evaluando una serie de proposiciones sobre estas relaciones y proporcionar un resumen para comentar sus implicancias. Una vez determinado los factores principales del proceso de vinculación, nos permitirá ver que el modelo es apoyado por parte de la literatura y contribuirá a la generación de conocimiento.

\section{Marco Teórico}

Las investigaciones sobre la relación de las empresas con las universidades en Latinoamérica vienen siendo estudiadas por investigadores como Alvarado-Borrego (2000), Siqueira et al (2009), López, Martínez, Medellín, Scanlon, \& Solleiro, (1994), entre otros, quienes buscan explicar los factores que reconocen las empresas como más importantes para vincularse a la universidad tradicional. Los estudios acerca de la relación entre la Empresa y la Universidad que cuenta con Programa Dual es escasa, en países como Alemania, Suiza, Austria, existe una buena cantidad de estudios acerca de este tipo de relaciones, mientras que en Latinoamérica la institución que viene profundizando este tipo de relaciones es la DHLA (2018), la cual ha puesto mucho empeño en contribuir con este tema, al igual que las Cámaras de Industria y Comercio Alemana ubicadas en países de Latinoamérica y las Universidades de la RED DHLA.

\subsection{Antecedentes}


La Formación Dual fue creada en Alemania bajo La Ley de Formación Profesional Berufsbildungsgesetz en alemán-, promulgada en 1968 y modificada en 2005 . Rego, Barreira, \& Rial (2015)

"En muchos países, la brecha entre colegio y universidad lleva al mismo tiempo a un alto nivel de desempleo juvenil y a una falta de personal técnico, lo que implica una carga para la economía y un freno al desarrollo de las empresas. En respuesta a ello Alemania ha desarrollado un sistema de formación profesional dual que combina las instrucciones en colegios vocacionales y academias con el aprendizaje práctico en las empresas" Bock (2018). La Formación Profesional en Alemania bajo el enfoque del Programa Dual es reconocida a nivel internacional, incluso la OCDE en un estudio comparativo con otros programas de escala internacional destacó el Programa Dual y su sistema de formación. Las razones para este reconocimiento se centraron en el alto nivel de Alemania en el empleo juvenil, la alta calidad de formación y la fácil movilidad hacia el mercado. Como característica del programa se ha identificado dentro de su funcionamiento cinco principios que le han permitido alcanzar este reconocimiento:

1. Cooperación entre agentes sociales, este principio promueve la integración y participación de los actores sociales junto a la empresa, de esta manera la empresa garantiza las condiciones básicas para la formación profesional pasando por el diseño y la actualización constante de los planes de estudio e incluso el financiamiento.

2. Aprendizaje en el proceso laboral, este principio se basa en el aprendizaje de las competencias que requieren los practicantes para el mejor desempeño en su proceso laboral. El resultado de instruir a los practicantes en las actividades laborales dentro de la empresa ha logrado desarrollar las capacidades necesarias de los aprendices o practicantes y garantizar la aplicabilidad de lo aprendido, por lo que responden a los intereses de las empresas y las universidades.

3. Aceptación de estándares nacionales. Al adaptarse este programa a nivel internacional, este debe cumplir con los estándares de cada país y de la institución que los adopte, el circuito es el mismo en todo los casos pasar por 4 fases de formación de acuerdo a los intereses de la empresa (comercial, operativo, financiero y de recursos humanos) al finalizar los estudiantes obtienen un certificado que valida sus competencias y logros alcanzados a través de su desempeño en la empresa.

4. Formación a cargo de personal capacitado. Otro principio que asegura la calidad de la formación y el impulso de los procesos de transformación en la empresa es que las universidades que forman a los practicantes cuentes con docentes formados en el modelo de formación dual y lo mismo debe suceder en la empresa, que los responsables a cargo del practicante entiendan el funcionamiento del modelo dual así como el correcto uso de las herramientas para la evaluación y formación del practicante en su fase práctica.

5. Investigación y asesoría institucionalizada en el área de formación profesional. Este principio busca mejorar permanentemente la formación profesional, y brindar la 
información más precisa durante la asesoría previa a la elección de la futura profesión. DHLA (2018).

El modelo de Formación Dual si bien es exitoso, aún sigue teniendo ciertas limitaciones parar replicarse, a pesar de ser un programa altamente alabado, los países que deciden importar el modelo deben pasar por un proceso adaptativo previamente, lo mismo sucede a nivel de empresas. Berstelmann Stiftung (2013)

Entre otras propuestas que explican la relación entre la Empresa y la Universidad podemos mencionar el Triángulo de Sábato, este modelo propone que una coordinación política entre la universidad, la empresa e inclusive el gobierno, actores económico que son necesarios para desarrollar capacidades técnicas y científicas Sabato \& Botana (1968). Otra propuesta más reciente es el modelo de la triple hélice que plantea tres versiones de vinculación. La primera, similar al triángulo de Sábato, funciona con políticas de gobierno que promuevan la alianza entre universidad-empresa. La segunda, señala que cada institución mantiene autonomía y se ven limitadas por las barreras que impone cada una de ellas. Finalmente, la tercera, son las llamadas Organizaciones Híbridas creadas a través de infraestructura para la generación de nuevo conocimiento Etzkowitz \& Leydesdorff (2000).

Cada uno de los modelos mencionado promueven el desarrollo económico de los países y en general ese desarrollo sería el resultado de la disminución del desempleo juvenil y el desarrollo y crecimiento empresarial. Para ello se requiere determinar los factores que los empresarios adoptan como importante para lograr esta vinculación. El estudio se centra en la relación empresa universidad por lo tanto el vínculo generado contribuye a la transformación interna de cada institución. Esta transformación se representa en las empresas por lograr contar con programas de transferencia de conocimiento eficientes, se ha valorado la capacitación técnica de su recurso humano y formación de sus directivos y mejora de la actitud ante la innovación Fernández et al. (2000).

En el caso de las universidades se manifiesta por alinear sus planes de estudio a una realidad empresarial y saber que logran captar y formar personal competente que contribuyan al desarrollo del país.

\subsection{Experiencias de la relación Empresa con la Universidad en Europa}

En cuanto a la experiencia del Reino Unido, se examinaron los factores que explican la influencia de las universidades en las actividades innovadoras en las empresas obtenidas por la transferencia de conocimiento. Para el análisis, realizaron encuestas cuya data fue clasificada en doce tipos de industrias, y por regiones para observar las características demográficas de la economía del país. Como resultado, identificaron la importancia de las universidades como fuente de conocimiento para la innovación de las empresas, destacando sobre otras posibles fuentes. La principal conclusión señala que los factores estructurales como la intensidad de I+D, el tamaño de la empresa y el entorno industrial son importantes para explicar que tan proclives son las empresas para utilizar las universidades en sus actividades de innovación Laursen \& Salter (2004).

Respecto a la intensidad del $\mathrm{I}+\mathrm{D}$, en España se analizaron los acuerdos de cooperación de $\mathrm{I}+\mathrm{D}$ en los sectores de fabricación y servicios respecto a la intensidad tecnológica. Los resultados señalan que la relación empresa con la universidad está relacionada con las características del sector y de la empresa. Para promover la cooperación y la innovación en las empresas es también importante el 
apoyo de la administración pública, sobre todo si se tratan de pymes. Asimismo, determinaron que las empresas tienen mayor interacción con las universidades españolas que con importantes universidades de la Unión Europea (UE), lo que limita lograr mayores beneficios y vínculos de cooperación. Esto sucede porque la financiación proviene de España y por tanto se espera a que la cooperación sea con universidades del país, ocurriría lo contraria si la financiación proviniese de la UE. Por ello, recomiendan unirse a programas de investigación de la UE para lograr mayores innovaciones y cooperación internacional Segarra-Blasco \& Arauzo-Carod (2008).

Otro estudio en España, desarrollaron el Modelo Satisfacción-CompromisoParticipación que señala la "satisfacción global de las empresas, compromiso percibido de las universidades, compromiso y participación de las empresas" como variables de colaboración de la relación empresa con la universidad. En el estudio, la información fue obtenida a través de encuestas dirigidas al director general de las empresas de la Comunidad Autónoma de Aragón. La mayoría de las empresas pertenecían a los sectores de servicios $(59,7 \%)$ y manufacturero $(38,8 \%)$. Asimismo, el $61,5 \%$ de las empresas eran pequeñas ya que contaban con menos de 50 empleados. Para analizar estas variables aplicaron modelos de ecuaciones estructurales en la metodología para identificar "variables latentes" que son menos perceptibles de observar y analizar su influencia sobre otras variables. Con ello, se determinó que el impacto de la relación empresa con la universidad es positivo en los ingresos económicos, en los recursos humanos, en la transferencia de conocimiento y la producción de investigación científica.
En Austria, la transferencia de conocimiento para el proceso de innovación industrial debe cumplir con dos objetivos. El primero asegurar la eficiencia de la investigación para cubrir habilidades, reducir costos y riesgos y explotar sinergias; y el segundo, para obtener acceso a oportunidades científicas y técnicas con investigadores de alta calidad, redes de investigación, instrumentos, nuevos conocimientos, entre otros. Klofsten \& Jones-Evans (2000).

\subsection{La relación Empresa con la Universidad en Estados Unidos y Canadá}

La encuesta nacional de la transferencia tecnológica universitaria en 115 instituciones de los Estados Unidos fue enfocada en las características de la organización; financiación y gestión; la relación entre las medidas de organización y gestión; y el rendimiento percibido. El análisis de la información sugiere la incidencia de diferentes tipos de mecanismos más innovadores y emprendedores en la transferencia tecnológica en las universidades estadounidenses. Además, se encontró una correlación entre el "rendimiento percibido de la unidad de transferencia tecnológica y las variables individuales y gerenciales como los años de experiencia, la orientación técnica y la frecuencia de la comunicación gerencial". Por ello, sugieren mayor investigación en el diseño y administración de transferencia tecnológica universitaria que aseguren el éxito de la relación empresa con la universidad Dill (1995).

En un estudio más reciente que analiza la interacción entre empresas manufactureras y universidades en Canadá, afirma que la probabilidad de colaboración entre la empresa con la universidad tiene que ver con el tamaño de la empresa y la 
importancia de la I+D en su sector, las empresas que buscan expandirse a nuevos mercados como estrategia de éxito, el desarrollo de los recursos humanos, entre otros. Las investigaciones de las universidades complementan las actividades en I+D de las empresas, ya que acceden a la investigación y desarrollan competencias con mayor facilidad, lo que ayuda a mejorar el rendimiento de la innovación y lograr mayor nivel tecnológico para las empresas. Hanel \& St-Pierre (2006)

\subsection{La relación Empresa con la}

\section{Universidad en Latinoamérica}

Al igual que en otras latitudes, Latinoamérica sufre de una brecha educativa alta, y esto obliga a muchos países de la región a contar con limitada mano de obra especializada, Como respuesta a esta realidad de brindar a las empresas el recurso necesario para sostener y mejorar su competitividad empresarial se tiene la experiencia de la Red Duale Hochschule Latinoamérica (DHLA) con presencia en seis países: Perú, México, Colombia, Ecuador y Costa Rica y Brasil, con doce universidades asociadas ${ }^{1}$. DHLA (2018)

El programa Dual de intervención está basado en el modelo innovador alemán de combinar la teoría con la práctica para garantizar la formación profesional y la eficiencia económica y empresarial, a través de "objetivos económicos, sociales e

${ }^{1}$ Perú. Univ. de San Martín de Porres. México. Univ. Interamericana para el Desarrollo. Colombia. Fundación Universitaria Empresarial de la Cámara de Comercio de Bogotá, Univ. Autónoma de Occidente de Cali, Univ. Autónoma de Bucaramanga, Corporación Universitaria Empresarial Alexander von Humboldt. Ecuador. Univ. de Cuenca, Univ. Católica Santiago de Guayaquil, La Escuela Superior Politécnica del Litoral, Universidad de las Américas, Costa Rica. Univ. Invenio, Brasil Univ. Asociación Educacional Dom Bosco. individuales". Así, bajo el modelo propuesto, los estudiantes de pregrado de las universidades adquieren conocimientos teóricos, para aplicarlos en las empresas asociadas de manera práctica, lo cual incrementa su motivación y experiencia laboral, y mejoran sus competencias profesionales.

Las ventajas para las empresas se traducen en beneficios económicos como, la reducción de costos en la adaptación laboral del personal nuevo, la ejecución de proyectos y solución de problemas, aseguramiento de personal calificado.

En México, Alvarado-Borrego (2000). realizaron un estudio siguiendo un análisis cualitativo de tipo explicativo sobre la relación de la Empresa con la Universidad y como esta contribuye al desarrollo regional, se concluye que la Universidad es el eje principal de cualquier sociedad y es necesario fortalecerla y reflexionar sobre el sistema educativo en general, además de su labor para formar ciudadanos para un determinado empleo, la relación de los sistemas educativo $\mathrm{y}$ productivo sirve para complementar las políticas de desarrollo social y económico en los sectores involucrados.

En las empresas, las universidades cumplen un doble rol, el de sustituir y complementar la investigación y desarrollo (I+D). El rol sustitutivo se cumple en empresas que no desarrollan $\mathrm{I}+\mathrm{D}$ y aquellas que subcontratan investigaciones que no pueden realizar en sus laboratorios. Mientras que el rol complementario se cumple en empresas grandes que tienen mayores recursos económicos e infraestructura. Asimismo, el papel de la universidad tiene mayor importancia en las empresas con mediano o bajo nivel tecnológico. Por ello, debe valorarse a las universidades no solo como apoyo para consultas o actividades técnicas, sino para el desarrollo de 
conocimiento, conferencias, investigaciones y otras interacciones de índole académico. Siqueira et al (2009).

En un estudio similar realizado en México, se exploraron las actitudes de 31 académicos de la Universidad Nacional Autónoma de México (UNAM) y 28 empresarios mexicanos frente a la experiencia de la RUE. Para los académicos, las principales motivaciones eran "ampliar perspectivas profesionales, mantenerse actualizado, aumentar el prestigio individual, aumentar el prestigio institucional y contribuir a la difusión del conocimiento. Para las empresas, las motivaciones eran "la necesidad de competir en los mercados internacionales, cubrir la falta de recursos humanos, instalar laboratorios internos de Investigación, reconocimiento de la universidad como una fuente importante de conocimiento explotable, conciencia de la importancia de aplicar la tecnología desarrollada en el país, percepción de que se está produciendo un cambio en las concepciones universitarias sobre ciencia, tecnología y cooperación con la industria”.

Sin embargo, nuevamente se encontraban limitaciones como "la brecha tecnológica entre el departamento de investigación de la universidad y el departamento de producción de la empresa, la falta de participación de las empresas en las primeras etapas de los proyectos y los desacuerdos relacionados con los costos, la confidencialidad y los derechos de propiedad" López, Martínez, Medellín, Scanlon, \& Solleiro (1994).

Los estudios previos evidencian diversos factores que influyen directa o indirectamente en las relaciones entre la Empresa con la Universidad, por ello es importante seguir investigando este tipo de motivaciones reflejados en los factores internos y factores externos de las empresas en su vinculación con las universidades, de esta manera proponer estrategias de vinculación tomando en cuenta la realidad empresarial. Para esta investigación se tomó como referencia los casos de empresas vinculadas a universidades que cuentan con Programa Dual. En la siguiente parte de la investigación se procede a entender los factores de análisis.

\subsection{Los Factores de Vinculación Empresa Universidad}

En este segmento se propone dos frentes los factores internos y factores externos que las empresas reconocen como importantes para vincularse a una universidad que cuenta con Programa Dual, además, se propone dos variables mediadoras que podrían explicar mejor esa relación de vinculación.

\subsubsection{Factores internos de la empresa}

Se consideran en el estudio dos tipos de factores de primer orden que las empresas toman en cuenta para vincularse con las universidades, los potenciales recursos humanos para la transferencia de conocimiento y el impacto económico de proyectos desarrollados.

\subsubsection{Potenciales recursos humanos para} transferencia de conocimiento

Identificar o contar con canteras donde se pueda tener potenciales recursos humanos se convierte en una estrategia que algunas empresas adoptan. Entre las principales características que buscan las empresas de sus recursos humanos es que estos cuenten con ciertas destrezas y habilidades (soft skill y hard skill) además de demostrar la facilidad para adaptarse a los cambios del entorno brindando respuesta inmediata a los requerimientos empresariales. 
Howells, Ramlogan, \& Cheng (2012) Sugieren que estar a la vanguardia del requerimiento de las empresas en temas de conocimiento es una tarea que debe recaer en instituciones serias que acompañen muy de cerca a las empresas. La formación del conocimiento dentro de las universidades debe contar con teoría actualizada y alineada a las nuevas tecnologías empleadas por los diversos sectores empresariales, entonces, se valora el conocimiento que adquiere un estudiante universitario durante su formación académica el cual al ingresar a una empresas será un activo valioso y apreciado, incluso llegando a ser un capital humano de alta calidad Kornhauser (2001), Gupta \& Singhal (1993) Bozeman, Fay, \& Slade (2013).

El recurso humano que se incorpora a la empresa es considerado de alta calidad, lo que facilita la continuidad de los procesos, su mejoramiento y la innovación, alcanzar este propósito simplifica la transferencia de conocimiento a una nueva generación. La captación de potenciales recursos humanos es posible a través de varios mecanismos, siendo el reclutamiento de universitarios y las prácticas para estudiantes los principales mecanismos. Perkmann \& Walsh, (2007). La transferencia de conocimiento requiere que haya una verdadera apropiación del conocimiento, comprender la relación entre lo que se tiene y lo que se puede lograr, llevando ese conocimiento a un contexto más amplio de tal forma que su uso eficiente genere desarrollo y se pueda obtener beneficios del mismo.

La transferencia de conocimiento en el marco de redes de cooperación ha venido creciendo debido al proceso interactivo interno y externo de las organizaciones, en los procesos interactúan diferentes tipos de conocimiento, se combinan distintas capacidades y recursos organizacionales
Los flujos de conocimiento, como lo señala Etzkowitz \& Leydesdorff (2000) permiten el intercambio de información para producir innovaciones en el marco de relaciones de cooperación a nivel territorial y global Etzkowitz \& Leydesdorff (2000), Cooke (2001).

Se mencionan algunas características para determinar los potenciales recursos humanos para la transferencia de conocimiento, entre ellos tememos.

\section{a) Habilidades y competencias}

Respecto a las habilidades y competencias se considera que es el "saber hacer en un contexto", que no solo se refiere a emplear ciertos instrumentos en una determinada situación, sino, estas vienen acompañadas de conocimientos, potencialidades, habilidades, destrezas, prácticas y acciones de diversa índole, estas acciones pueden ser de tipo personales, colectivas, afectivas, sociales, culturales. Irigoyen, Jiménez, \& Acuña (2011)

\section{b) Ideas innovadoras}

La innovación genera posiciones encontradas, por lo cual es necesario que en el proceso se cuente con personal que se adapte rápidamente al cambio y contribuya en la sensibilización de la importancia de estas acciones. Coleman (2003). Link \& Siegel (2007) Mencionan qué en una organización, las habilidades del capital humano juegan un rol importante en la implementación de procesos innovadores. Los empresarios requieren trabajadores preparados para llevar el conocimiento a la práctica, que tenga espíritu emprendedor, sean innovadores y que se adapten a los cambios. Simanaviciene, Zuzeviciute, \& Praneviciene (2017).

Entonces la innovación y creatividad son algunas características que se espera del 
nuevo personal que se incorpora a la empresa, para ello es necesario que las universidades propongan diversas metodologías que permitan a sus estudiantes explorar y sostener estructuradamente una propuesta innovadora y creativa. Reddy, (2011), Waples \& Ropella (2003).

\section{c) Personal ávido de adquirir conocimiento}

El personal de la empresa debe contar con aptitudes y actitudes para la aceptación de nuevo conocimiento y la generación del mismo. Pasar de un aprendizaje teórico a una realidad práctica muchas veces requiere de la predisposición del aprendiz para lograr aprender en tiempo reducidos lo requerido en los puestos de labor. Como mecanismos de respuesta algunas empresas se preocupan en brindar al personal los conocimientos necesarios que les permitan mejorar sus relaciones interpersonales e intrapersonales de esta manera al momento de adquirir nuevo conocimiento cuentan con las bases necesarias para lograr una mejor recepción de la información.

\section{d) Desarrollo de investigaciones}

Teniendo en cuenta las necesidades del sector empresarial, se hace imperativo fundamentar una relación bidireccional donde los participantes (empresa - universidad) se complementan en el proceso para un mejor entendimiento y apreciación del contexto y lograr una solución científica y tecnológica derivada de la investigación académica.

El modelo tradicional de transferencia de conocimiento se fundamenta en una visión lineal, unidireccional y en el que las patentes son el medio principal de transferencia. Frente a este patrón "tradicional" se viene abriendo paso un patrón relacional de transferencia de conocimiento basado en la cooperación y la interacción continua entre investigadores tanto universitarios como de organizaciones no-académicas Perkmann (2013).

e) La retroalimentación de los universitarios sobre los resultados de las investigaciones

Las empresas puedan incrementar su conocimiento e identificar nuevas investigaciones de innovación; explotar el conocimiento nuevo o existente para crear nuevos productos o procesos o reducir sus costos; mejorar las capacidades de resolución de problemas de la empresa al contratar graduados calificados y capacitando al personal de la empresa para interpretar y aplicar los conocimientos en toda la organización Bishop, D’Este, \& Neely (2011). Es necesario el acompañamiento por parte de los miembros de la universidad para exponer los resultados de investigación y los beneficios de los hallazgos encontrados que favorecen a las empresas.

Por su parte D'Este, García Quevedo, \& Mas-Verdú (2014) señalan que una estructura de incentivos excesivos provocan efectos no deseados en la cultura investigativa abierta, los cuales se reflejan en tres aspectos: primero, se limita la posibilidad de compartir y difundir los resultados de las investigaciones como corresponde a la comunidad científica; segundo, se sesgan las investigaciones a intereses particulares en detrimento de una investigación de carácter más fundamental o básica; y finalmente, se crea un exceso de celo en la protección de los derechos de propiedad intelectual generando contrapunteos entre las universidades y las empresas al punto que se dificulta la disposición de colaborar.

\section{f) Alto nivel académico}

Las empresas buscan crear relaciones de cooperación con universidades de alto prestigio y la colaboración de sus miembros que aporten en la solución de sus problemas y 
alcance de nuevas oportunidades, el logro de esta cooperación se evidencia por los resultados alcanzados después de una experiencia ya sea por la ejecución de proyectos en conjunto entre empresa y la universidad o el desempeño del personal incorporado en la empresa. En ambos casos se reconoce que los participantes involucrados contaron con un alto nivel académico demostrado.

\subsubsection{Impacto Económico por el desarrollo de proyectos}

Las empresas buscan constantemente optimizar sus procesos, mejorar su productividad, reducir sus costos e incrementar su participación en el mercado. La investigación y el conocimiento que provee la universidad ofrece nuevas oportunidades para que las empresas agreguen valor a sus procesos y maximicen el potencial de crecimiento económico Kenney (1986); Slaughter \& Leslie (1997) Slaughter \& Rhoades (2004).

La mayoría de empresas grandes cuentan con un plan estratégico desarrollado, el cual cuenta con objetivos estratégicos definidos, los mismo que han sido propuestos para cerrar brechas identificadas, y obtener beneficios como el crecimiento del negocio y mejorar la reputación de la organización Mead et al (1999).

Las Pymes en algunos casos tienen limitaciones para contar con un plan estratégico, las acciones que ejecutan por lo general son de corto plazo de acuerdo al entorno donde se desempeñan, en ese escenario contar con la colaboración de una universidad que le brinde un acompañamiento le proporciona metodología, le permite alinear objetivos, mejora la confianza en la toma de sus decisiones, desarrolla una comunicación efectiva y fortalece su postura analítica como parte del trabajo colaborativo que desarrollan. Mead. (1999); Bonaccorsi \& Piccaluga (1994).

La intervención de la universidad en un proyecto de la empresa debe ser capaz de generar ganancias, a partir de reconocer pérdidas por errores en sus procesos y de esta manera proponer el desarrollo de proyectos que generen valor Bloedon \& Stokes (1994); Cohen et al (2002). Con la vinculación empresa - universidad, se forman redes entre académicos y empresarios, que desarrollan contactos estrechos y utilizan esta red como fuente de ideas para nuevos proyectos de investigación Mansfield (1995). La eficiencia de la vinculación puede reflejarse cuando "produce más beneficios por un costo dado o, cuando genera menos costos por un beneficio dado" Landry \& Amara (1998).

Entonces de acuerdo a lo antes mencionado se propone las siguientes hipótesis:

- H1. Los factores internos son determinantes en la vinculación empresa - universidad

- H1a. Los potenciales recursos humanos para transferir conocimiento que provee la universidad son determinantes para la vinculación con la empresa

- H1b. El impacto económico generado por el desarrollo de proyectos donde la universidad participa son determinantes para la vinculación con la empresa

\subsubsection{Factores externos}

Se consideran en el estudio tres tipos de factores externos las capacitaciones, la influencia del estado política, social y económica y el posicionamiento.

\subsubsection{Capacitaciones}

Las capacitaciones que brindan las empresas a sus colaboradores cumplen un rol para 
maximizar el desarrollo de las competencias. Aunque es importante y reconocida por la empresa, las capacitaciones necesariamente no se adecuan a sus necesidades empresariales o temas relacionados a sus actividades operativas que les permita solucionar sus propios problemas.

En las instituciones con Programa Dual, es imperativo brindar la capacitación a los responsables de los practicantes que estarán cumpliendo labor en la empresa. La capacitación recibe el nombre de Formación de Instructores (FDI) y se brinda a través de las universidades miembros de la RED DHLA en cooperación con las Cámaras Alemanas del país correspondiente. el objetivo es que el personal de la empresa a cargo de aprendices pueda ser capaz de elaborar un plan de formación, seguimiento y control a través de la aplicación de metodologías diseñadas específicamente para esta labor, este tipo de entrenamiento luego pueden ser replicadas en otros puestos de la empresa.

Los instructores empresariales son responsables de planificar el contenido de los programas de formación y gestionar la formación profesional que se lleva a cabo en la empresa. Los instructores deben estar cualificados desde el punto de vista profesional y deben dominar aquellas competencias que quieren enseñar a los aprendices. El instructor empresarial debe desempeñar diferentes roles en una misma figura: instructor, superior, educador, compañero de trabajo y experto. Dualvet Programa de Aprendizaje PermanenteComisión Europea (2015).

\subsubsection{Influencia del Estado, Política, Social y Económica}

La investigación presenta las posibles relaciones causa-efecto que puedan darse en la dinámica de interacción entre las naciones, estados, gobiernos y las instituciones de educación superior que hayan decidido desarrollar o estrechar vínculos con las organizaciones, a través del desarrollo y ejecución de Programas Dual. El Programa Dual Universitario parte de la premisa de formar a un estudiante, dentro de un programa de estudio específico, bajo los principios de la Dualidad, una universidad que tiene un vínculo de cooperación con una empresa, que puede pertenecer a cualquier sector y actividad económica, para fortalecer el capital humano en los territorios.

El modelo del Programa Dual, resulta beneficioso para los territorios que consideran que es el capital humano, la fuerza que subyace el desarrollo, por ello distintos gobiernos han implementado políticas para fortalecer la vinculación entre la organización y la universidad con el fin de lograr el desarrollo económico, apoyado en la investigación académica. DHLA (2018)

Esta relación de la empresa con la universidad impacta en aspectos socioeconómicos, culturales, educativos mediante el incentivo para la innovación tecnológica, desarrollo de nuevos productos, mejoramiento de técnicas, así como la aplicación y desarrollo de conceptos Arocena \& Sutz (2005).

El Programa Dual, no solo cumplen el objetivo de "formar ciudadanos productivos para un determinado empleo", sino para "complementar las políticas de desarrollo social y económico" de los diversos sectores de la economía de un país; y sería relevante agregarle el objetivo de formar seres humanos íntegros, comprometidos con la sustentabilidad del planeta y en preservar aquellos valores humanos que persigan simultáneamente el desarrollo social y colectivo, todos ellos, posiciones no solo válidas, sino claramente complementarias. Alvarado-Borrego (2000) 
En los países latinoamericanos, un proceso clave para el desarrollo de sus sectores industriales ha podido precisamente ubicarse en los mecanismos de vinculación entre la empresa con las universidades "el nivel de relacionamiento entre los agentes de los sistemas de innovación, el desarrollo propio de las industrias enfocadas hacia el desarrollo tecnológico y los niveles de inversión y desarrollo en Investigación y Desarrollo -I+D- se encuentran apalancados fuertemente por la cooperación entre las empresas y las universidades".

En ese aspecto se puede considerar algunos puntos tales como

\section{a) Cumplimiento de la política pública}

En su sentido más básico, una política es el marco de referencia que delimita o guía la toma decisiones, en términos de políticas públicas, se espera que documente el conocimiento sobre un tema puntual, propicie la acción o gestión y que permita la evaluación; el conocimiento para ser aplicado a problemas concretos; la acción para la gestión administrativa en función de un resultado social y la evaluación, para que permita la conjunción de una serie de mecanismos institucionales para analizar su eficacia y determine pautas para mejorar el futuro. Se puede afirmar que el cumplimiento de la política pública se trata de comparar los resultados de dichas declaraciones y los medios utilizados versus los objetivos propuestos y logros alcanzados.

"Son las políticas públicas una concatenación de actividades, decisiones o de medidas coherentes por lo menos en su intención, y tomadas principalmente por los actores del sistema político-administrativo de un país con la finalidad de resolver un problema colectivo". Larrue, (2000).

Al ser un estudio de Programas Duales en países de Latinoamérica se consideró para el estudio 4 países, donde las políticas laborales se ven lideradas por los Ministerios de Trabajo y Ministerio de Educación, quienes asumen el reto de preparar a los territorios para el desarrollo con programas de reentrenamiento que articulen la oferta de las universidades y las necesidades de las empresas.

En la tabla siguiente se describen las características del marco normativo y regulatorio por cada país miembro de la Red Duale Hochshule Latinoamérica.

Tabla 1. Legislación de Programa Dual Red DHLA

\begin{tabular}{|c|c|c|c|c|c|c|c|c|}
\hline \multirow[b]{2}{*}{$\begin{array}{l}\text { País de la } \\
\text { Red DHLA }\end{array}$} & \multirow[b]{2}{*}{$\begin{array}{c}\text { Legislación } \\
\text { Aprendices y } \\
\text { Prácticas }\end{array}$} & \multirow{2}{*}{$\begin{array}{c}\text { Compensación } \\
\text { SI }\end{array}$} & \multirow[b]{2}{*}{ Salud } & \multicolumn{2}{|c|}{ Seguridad Social } & \multirow[b]{2}{*}{$\begin{array}{c}\text { Vacaciones } \\
\text { pagas }\end{array}$} & \multicolumn{2}{|c|}{ Estadio en la empresa } \\
\hline & & & & Pensión & $\begin{array}{l}\text { Riesgos } \\
\text { laborales }\end{array}$ & & $\begin{array}{l}\text { Jornada } \\
\text { Horas x } \\
\text { Semana }\end{array}$ & $\begin{array}{c}\text { Temporalidad } \\
\text { de la } \\
\text { Vinculación }\end{array}$ \\
\hline Perú & $\begin{array}{l}\text { Ley } 28518 \text { del } \\
2005\end{array}$ & $x$ & SI & NO & NO & NO & 30 & $\begin{array}{l}2 \text { - 3,5 Años } \\
\text { Flexible* }\end{array}$ \\
\hline Colombia & $\begin{array}{l}\text { Decreto } 789 \text { del } \\
2002\end{array}$ & $x$ & SI & SI & SI & NO & $40-48$ & $\begin{array}{l}2 \text { - 3,5 Años } \\
\text { Flexible* }\end{array}$ \\
\hline México & $\begin{array}{l}\text { Ley Marco de } \\
\text { Prácticas }\end{array}$ & $x$ & NO & NO & NO & NO & 40 & $\begin{array}{c}2 \text { - 3,5 Años } \\
\text { Flexible* }\end{array}$ \\
\hline
\end{tabular}




Ley de pasantías
en el sector
Ecuador
organizacional.
RPC-SO-10-
No.129-2018

Fuente: Marco legal de acuerdo a países participantes en el estudio que cuentan con programa dual. Elaboración propia.

En los países citados existen marcos normativos que favorecen la contratación de los jóvenes, y en ciertos casos las organizaciones pueden obtener ciertos beneficios como reducción de gravámenes y tasas, compensación de puestos de trabajo y subvenciones económicas deducibles para efectos tributarios.

La vinculación y contratación de personal nuevo, supone un proceso previo de perfilación, experiencia y entrenamiento, en la que se considera la participación de jóvenes aprendices que estén monitoreados y con apoyo de universidades que se encuentren operando dentro del Programa Dual.

\section{b) Enfoque socialmente responsable}

El enfoque de responsabilidad social por su incipiente conocimiento y no clara definición en el impacto sobre el beneficio para las empresas ha llevado a generar posturas polarizadas, por un lado, aquellos basados en consideraciones de orden moral o filantrópico, con una abstracción de la realidad y por el otro, los centrados en el registro estadístico de variables e indicadores con una limitada interpretación adecuada de la realidad por su análisis individual de cada caso en la empresa que lo practica. Solis (2008)

Una práctica que las empresas con Programa Dual ejecutan es asegurar el relevo generacional de su fuerza de trabajo, las empresas contribuyen con el desarrollo académico y profesional de sus colaboradores. Es evidente que en países de
Latinoamérica existe una falta de cualificación de personal para incorporar a las empresas. Por ello brindar la oportunidad a más jóvenes a ser incluidos en la vida laboral y así disminuir el desempleo es uno de los grandes problemas sociales a superar Kupffer (2005), ese accionar podría estar considerada como una práctica de responsabilidad social. Programas Dual vinculan los esfuerzos de las empresas y las universidades para mejorar la empleabilidad de sus estudiantes y graduados, preparándolos con las habilidades que las organizaciones demandan y ser más empleables.

\subsubsection{Posicionamiento}

Kotler (2008), define el posicionamiento en el mercado como el resultado de lograr que un producto ocupe un lugar claro, distintivo y deseable con relación a los competidores, en la mente de los compradores o consumidores del grupo objetivo.

El posicionamiento es un concepto de gestión que viene ganando espacio en instituciones educativas en todo el mundo gracias al aumento de la competencia nacional e internacional. Las universidades de todo el mundo han comenzado a buscar una estrategia de diferenciación que las haga únicas y les permita atraer estudiantes, personal académico de calidad y vinculación a empresas de prestigio, públicas y privadas.

La educación superior practica un nuevo vocabulario, que viene de las grandes empresas del sector privado, como gerencia de marca, comunicaciones corporativas, identidad y reputación, haciendo que las 
organizaciones de educación superior sean más conscientes del vínculo entre lo que representan en términos de valores $y$ características del producto educativo y la forma como son percibidas Wæraas (2009).

El posicionamiento de la marca de una universidad es la efectividad del producto y las percepciones acerca del mismo, para lo cual es importante evaluar la calidad educativa, en donde los estudiantes cumplen un papel importante dentro de este proceso. Por lo tanto, los resultados de la educación superior, dependen en gran medida de las habilidades, adquiridas y demostradas por los propios estudiantes, individualmente y como grupo, Temple (2006).

Estas habilidades son reconocidas por las empresas y funcionan como un referente de vinculación entre la empresa y la universidad. Las universidades fortalecen constantemente el desarrollo de su producto, preparan estudiantes cada vez más competitivos, acorde con la realidad empresarial, se busca permanentemente que las intervenciones de los estudiantes en las empresas sean más destacadas, por lo cual se implementa estrategias para mantener $\mathrm{y}$ mejorar logros que destaquen una posición competitiva. Estas ventajas diferenciadoras deben ser comunicadas por las universidades de manera efectiva y consistente a todos los públicos de interés Wæraas (2009).

Por un lado, el posicionamiento de una universidad está ligado directamente a la gestión de la reputación y sus relaciones públicas, implica intervenir para cambiar las cosas con el fin de lograr una mejor enseñanza, investigación e instalaciones físicas Temple (2006). La cooperación con la empresa, ayuda a la universidad a mejorar su programa académico, y emplear nuevas maneras de capacitar a sus estudiantes.

Por otro lado, algunas empresas valoran la ubicación geográfica de las universidades y su proximidad a la empresa, en un entorno donde los tiempos de traslado no generan valor, el impacto de la distancia geográfica ha sido ampliamente discutido, revelando que las distancias cortas facilitan las interacciones presenciales que fomentan la transferencia de conocimiento y la innovación Antonelli (2000).

Entonces de acuerdo a lo antes mencionado se propone las siguientes hipótesis:

- H2. Los factores externos son determinantes en la vinculación empresa - universidad

- H2a. Las capacitaciones que brindan las universidades son determinantes para la vinculación con la empresa

- H2b. La influencia del estado política, social y económica son determinantes para la vinculación con la empresa

- H2c. El posicionamiento que tiene la universidad es un determinante para la vinculación con la empresa

\subsubsection{Variables mediadoras}

En el estudio se considerado dos variables mediadoras que pueden influenciar como determinantes de los factores internos y factores externos, estas son; Participación de los representantes de la empresa en actividades académicas y Los costos en la formación de recursos humanos para transferencia de conocimiento.

\subsubsection{Participación de los representantes de la empresa en actividades académicas del Programa dual}

La difusión de conocimientos a través de seminarios, talleres o cursos dictados por académicos y/o empresarios sirve para mantener una relación a largo plazo en la vinculación. Bloedon \& Stokes, (1994), éste 
acercamiento debe estar complementado con un sistema de comunicación adecuado que consolide la relación entre los socios y el éxito del vínculo Bonaccorsi \& Piccaluga (1994); Chisholm (996); Child \& Faulkner (1998). Inzelt, (2004). Para Kharazmi (2006). La participación de representantes de las empresas en los Programas Dual se practica cuando los representantes se involucran en el diseño de los planes de estudio o como expositores en eventos académicos.

Entonces de acuerdo a lo antes mencionado se propone la siguiente hipótesis:

- H3. La participación de los representantes de la empresas en actividades académicas incrementa la relación entre los factores y la vinculación empresa - universidad

\subsubsection{Costos de selección y formación de personal}

En el caso de las empresas que participan en el Programa Dual de la Red DHLA, las pymes principalmente les ha resultado atractivo participar con Universidades con Programa Dual, porque son las Universidades las que asumen los costos de formación del estudiante, las asesorías, el seguimiento y control de los procesos y proyectos.

Un ejemplo se presenta en la selección de personal, las Pymes al estar vinculadas a la Universidad, tienen la posibilidad de elegir estudiantes que ya cuentan con ciertas habilidades desarrolladas que ellos necesitan, el logro de alcanzar resultados idóneos en una organización depende en muchos casos de contar con personas idóneas y que estos estén ubicados en puestos idóneos, "la persona idónea para el puesto idóneo", este pensamiento permitiría reducir la incertidumbre en cuanto a la selección y reducir la rotación de personal, la misma que llevado a términos económicos se podría traducir en pérdidas en el proceso de selección de personal. Peña, (2005).

Incorporar estudiantes universitarios a la empresa beneficia la reducción de costos de capacitación y rotación de personal, contribuyendo a la retención de talentos a largo plazo. Waples (2003).

Entonces de acuerdo a lo antes mencionado se propone la siguiente hipótesis:

- H4. Los costos de formación de recursos humanos incrementa la relación entre los factores y la vinculación empresa - universidad

\subsubsection{Vinculación Empresa con la Universidad}

Respecto a la variable vinculación, su operacionalización propone que se identifique tres elementos, primero haber alcanzado un nivel alto de satisfacción en la experiencia, segundo tener la intención de continuar con la experiencia y tercero estar dispuestos a recomendar la experiencia a otras empresas.

\section{Método}

Para el desarrollo del estudio se aplicó Ecuaciones Estructurales con Mínimos Cuadrados Parciales (SEM- PLS). Se identificó a las universidades miembros de la red DHLA que tienen Programa Dual activo brinden información sobre las empresas que participaron en el periodo 2017- 2018. Las universidades que finalmente formaron parte del estudio fueron: La Universidad San Martin de Porres (Perú), Universidad de Cuenca (Ecuador), Universidad Interamericana para el Desarrollo (México), Corporación Universitaria Empresarial Alexander von Humboldt, Universidad Autónoma de Bucaramanga, Fundación Universitaria Empresarial de la Cámara de 
Comercio de Bogotá y la Universidad Autónoma de Occidente (Colombia). El total de empresas reportadas por estas instituciones fueron 836 .

\subsection{Muestra}

Se aplicó el cálculo de muestra para poblaciones finitas y obtuvo un tamaño de muestra esperado de 263. Sin embargo, como resultado de la recolección final de empresas encuestadas se alcanzaron a 294 empresas; encuestándose a los directivos vinculados con la gestión del Programa Dual.

La muestra final estuvo compuesta por empresas pequeñas $(50 \%)$, medianas $(21 \%)$ y grandes (29\%). Además, pertenecían a los sectores manufactura (16\%), comercio (23\%), servicio (47\%) y otros sectores (14\%). La descripción se muestra en la siguiente tabla.

Tabla 2. Muestra de empresas según país y tamaño

\begin{tabular}{ccccc}
\hline País & N $^{\circ}$ de Empresas & $\begin{array}{c}\text { Porcentaje } \\
\text { participación }\end{array}$ & Muestras Esperadas & $\begin{array}{c}\text { Muestras } \\
\text { alcanzadas }\end{array}$ \\
\hline Perú & 24 & $3 \%$ & 8 & 17 \\
Ecuador & 66 & $8 \%$ & 21 & 47 \\
México & 369 & $44 \%$ & 116 & 83 \\
Colombia & 377 & $45 \%$ & 119 & 147 \\
Total & 836 & $100 \%$ & 263 & 294 \\
\hline
\end{tabular}

Fuente: Empresas vinculadas al Programa Dual de las universidades miembros de la Red DHLA, periodo 2017 -2018. Elaboración propia

\subsection{Medición y Variables}

Escala de factores internos $y$ externos asociados a la vinculación de Universidades con Programa Dual

Se aplicó a los directivos de las empresas que participan el Programa Dual de la Red DHLA un cuestionario estructurado creado ad hoc para la presente investigación, validado por investigadores de 4 países. Los factores analizados se agruparon en dos grandes grupos: factores internos y externos. En ambos casos, los encuestados respondieron a una escala de 7 puntos: 7= Extremadamente importante, 6= Muy importante, 5= Moderadamente importante, 4= Ligeramente importante, 3= Poco importante, 2 = Mínimamente importante, $1=$ Para nada importante.
La escala de factores internos está compuesta por dos dimensiones:

a) Potenciales recursos humanos para la transferencia de conocimiento: formada por seis (06) ítems que por un lado exploran el aporte de los estudiantes a la organización en términos de innovación, participación especializada y capacidad de adquisición de conocimientos. Por otro lado, la escala recoge información sobre la oportunidadbeneficio de las empresas con relación al desarrollo de investigaciones y la retroalimentación producto de la implementación de proyectos. Todas estas condiciones aseguran la transferencia de 
conocimiento de la universidad hacia la empresa.

b) Impacto económico: formado por cuatro (4) ítems que exploran si las empresas vinculadas programa dual se benefician con el logro de sus objetivos estratégicos, la mejora de su productividad, la ejecución de nuevos proyectos y una mayor ratio de costo - beneficio.

La escala de factores externos también está conformada por tres dimensiones:

a. Capacitación: compuesta por dos (02) ítems que indagan beneficios en el ámbito de capacitación de personal logrados por su involucramiento en el Programa Dual.

b. Influencia del Estado, política, social y económica: Escala de cuatro (03) ítems que evalúan beneficios organizacionales vinculados al cumplimiento de políticas nacionales, la responsabilidad social y obtención de beneficios tributarios.

c. Posicionamiento: formado por tres (3) ítems que evalúan atributos que la empresa reconoce en la universidad y permiten diferenciarla de las demás universidades. Esta diferenciación también se relaciona a la recomendación recibida de otras empresas sobre los atributos de la universidad. Los ítems exploran las recomendaciones de otras empresas, la ubicación geográfica estratégica de la universidad y el marketing que esta emplea.

\subsection{Participación en el Programa Dual}

La escala explora el involucramiento de las empresas y su participación en actividades conjuntas con las universidades el marco del Programa Dual. Está formada por tres (03) ítems referidos a la participación en eventos académicos, reuniones empresariales $\mathrm{y}$ desarrollo de proyectos conjuntos. Los participantes reportan si han participado en estas actividades a través de una escala dicotómica de respuesta (Sí - No).

\subsection{Costos de selección y capacitación de personal}

La escala está conformada por dos (02) ítems que recogen información sobre la reducción de los costos en selección y capacitación del personal producto de la participación de las empresas en el Programa Dual. Asimismo, los encuestados respondieron a una escala de ordinal de 7 puntos: $7=$ Extremadamente importante, $6=$ Muy importante, $5=$ Moderadamente importante, 4= Ligeramente importante, 3= Poco importante, $2=$ Mínimamente importante, 1= Para nada importante.

\subsection{Vinculación con el Programa \\ Dual/Satisfacción-Compromiso}

Escala compuesta por tres (03) ítems y cuyo objetivo es valorar la vinculación de las empresas con el Programa Dual. La vinculación de las empresas es medida a través de indicadores relacionados a la intención de mantener la relación, la satisfacción alcanzada y la recomendación del programa a otras empresas.

\subsection{Análisis de datos}

Para evaluar el modelo propuesto se aplicó Ecuaciones Estructurales con Mínimos Cuadrados Parciales (SEM- PLS). En este sentido, los análisis del modelo de medida y 
estructural se realizaron con el paquete estadístico SmartPLS 3.2.3 Ringle, Wende, \& Becker (2017) Las propiedades de medida de las escalas se establecieron a través de evidencias de consistencia interna (fiabilidad) y validez de constructo. El impacto de los factores internos y externos sobre la vinculación de las empresas se evaluó considerando los siguientes índices: 1) los coeficientes beta estandarizados ( $\beta$ ) que muestran la relación entre los constructos. Se espera que su valor sea mayor a 0.1 para ser considerados como significativos; 2) el coeficiente de determinación $\left(\mathrm{R}^{2}\right)$, que muestra el porcentaje de varianza explicada de los factores internos y externos sobre la participación, reducción de costos y la vinculación con las Universidades con Programa Dual. El valor del coeficiente debe ser mayor a 0.1 para considerarse significativo Hair, Hult, Ringle, \& Sarstedt (2017); y 3) La técnica de Bootstraping, útil para establecer la precisión de los coeficientes de trayectoria (beta) y el contraste de hipótesis de significación de la prueba estadística (muestra $=5000$ veces; casos $=294$, utilizando la opción sin cambio de signo). De esta manera se estimó los errores estándar, los valores de $\mathrm{t}$ de student y el $\mathrm{p}$ value de los coeficientes de trayectoria $(\beta)$.

\section{Resultados}

\section{Fiabilidad y validez de las escalas}

\subsection{Fiabilidad}

Las escalas utilizadas mostraron adecuadas propiedades de medida. La fiabilidad fue evaluada mediante el análisis de la consistencia interna de los datos a través del coeficiente alfa de Cronbach, Omega y de fiabilidad compuesta (Hair, Ringle, Hult, \& Sarstedt (2014). En el caso de las dimensiones de los factores internos y externos, y las escalas de costos de RRHH y vinculación; todos los coeficientes oscilaron entre .697 y .967 , superando el valor corte de .708 Nunally \& Bernstein (1994). Además, se estableció la fiabilidad de los constructos de segundo orden. Los factores internos $(\mathrm{FC}=.910)$ y factores externos $\quad(\mathrm{FC}=.891)$ también cumplieron el criterio de consistencia interna. Una situación particular se observó en la escala de participación. Los coeficientes alfa de Cronbach y Omega se encuentran por debajo del punto de corte aceptado. Sin embargo, por el número de indicadores que conforman la escala el coeficiente de fiabilidad compuesta obtenido (.709) se admitió como evidencia suficiente de consistencia interna.

Tabla 3.Fiabilidad y Validez de Escalas

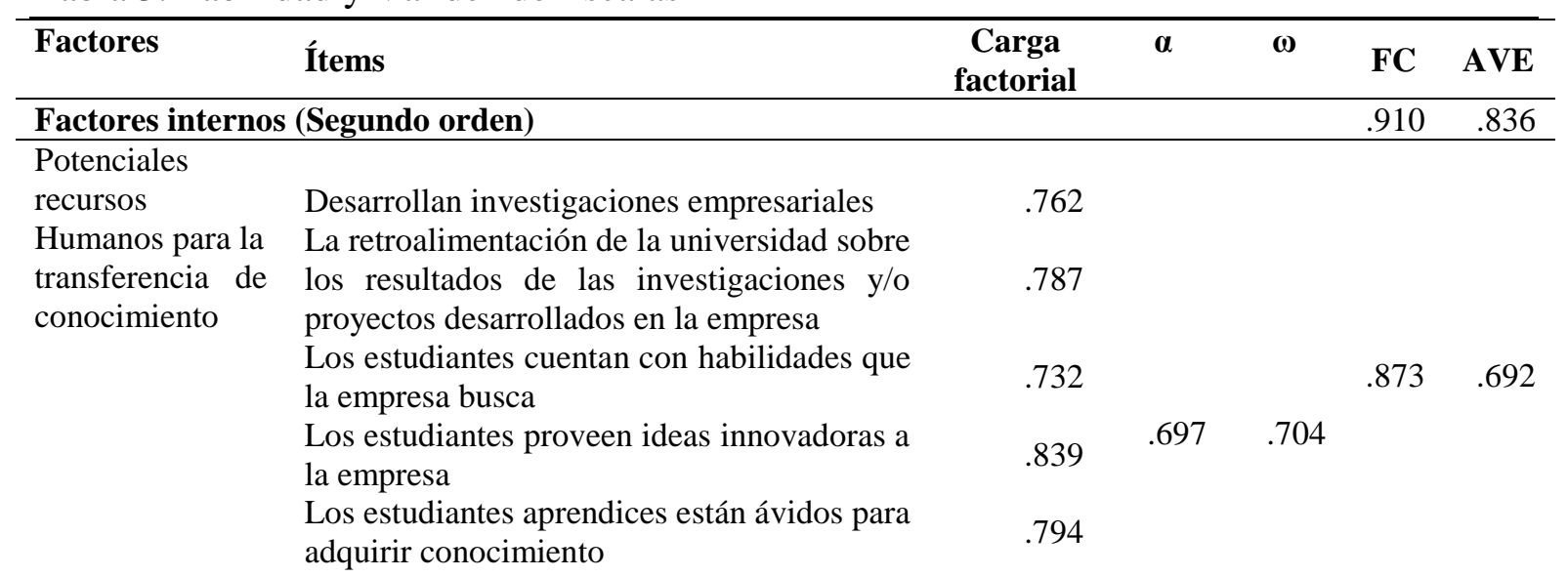


La formación de estudiantes en el sistema dual simplifica la formación de especialistas y futuros colaboradores

Económico Apoya en el cumplimiento de objetivos estratégicos

.850

Mejora el nivel de productividad en la empresa

La oportunidad para desarrollar nuevos proyectos en la empresa

El ratio de costo-beneficio positivo

.868

.720 $\begin{array}{lll}.849 & .859 & .899\end{array}$

.692

\begin{tabular}{|c|c|c|c|c|c|c|}
\hline \multicolumn{5}{|c|}{ Factores externos (Segundo orden) } & \multirow{4}{*}{$\begin{array}{l}.891 \\
.848\end{array}$} & \multirow{4}{*}{$\begin{array}{l}.804 \\
.650\end{array}$} \\
\hline Posicionamiento & Las recomendaciones de otras empresas & .767 & \multirow{3}{*}{.730} & \multirow{3}{*}{.731} & & \\
\hline & Su ubicación geográfica es estratégica & .746 & & & & \\
\hline & Emplea un marketing agresivo & .899 & & & & \\
\hline \multirow[t]{3}{*}{ Capacitaciones } & Brinda capacitaciones al personal de la & 813 & \multirow{3}{*}{.750} & \multirow{3}{*}{.751} & \multirow{3}{*}{.889} & \multirow{3}{*}{.800} \\
\hline & empresa & .015 & & & & \\
\hline & $\begin{array}{l}\text { Promueve la formación de instructores } \\
\text { empresariales }\end{array}$ & .771 & & & & \\
\hline \multirow{3}{*}{$\begin{array}{l}\text { Influencia de la } \\
\text { política social y } \\
\text { económica }\end{array}$} & La obtención de beneficios tributarios & .762 & \multirow{3}{*}{.717} & \multirow{3}{*}{.766} & \multirow{3}{*}{.842} & \multirow{3}{*}{.646} \\
\hline & $\begin{array}{l}\text { Cumplir con las políticas del estado de } \\
\text { forma constructiva o impositiva }\end{array}$ & .810 & & & & \\
\hline & $\begin{array}{l}\text { Cumplir con el compromiso socialmente } \\
\text { responsable }\end{array}$ & .810 & & & & \\
\hline \multirow[t]{3}{*}{ Participación } & Eventos académicos/exposiciones & .799 & \multirow{3}{*}{.408} & \multirow{3}{*}{.444} & \multirow{3}{*}{.709} & \multirow{3}{*}{.483} \\
\hline & $\begin{array}{ll}\text { Eventos } & \text { empresariales/desayunos } \\
\text { empresariales } & \end{array}$ & 686 & & & & \\
\hline & Proyectos conjuntos & .508 & & & & \\
\hline \multirow[t]{2}{*}{$\begin{array}{l}\text { Costos } \\
\text { RRHH }\end{array}$} & $\begin{array}{l}\text { Reducción de los costos de capacitación de } \\
\text { personal }\end{array}$ & .967 & \multirow{2}{*}{.931} & \multirow{2}{*}{.931} & \multirow[t]{2}{*}{967} & \multirow[t]{2}{*}{.935} \\
\hline & $\begin{array}{l}\text { Reducción de los costos de selección de } \\
\text { personal }\end{array}$ & .965 & & & & \\
\hline \multirow{3}{*}{$\begin{array}{l}\text { Vinculación } \\
\text { empresa- } \\
\text { universidad }\end{array}$} & $\begin{array}{l}\text { Satisfacción con la } \\
\text { Universidad-Empresa }\end{array}$ & .847 & \multirow{3}{*}{.844} & \multirow{3}{*}{.848} & \multirow{3}{*}{.905} & \multirow{3}{*}{.762} \\
\hline & Intención de mantener la vinculación & .848 & & & & \\
\hline & $\begin{array}{l}\text { Recomendación a otras empresas para } \\
\text { inicial la vinculación con la universidad }\end{array}$ & .909 & & & & \\
\hline
\end{tabular}

Nota: Alfa de Cronbach ( $\alpha$ ), Coeficiente omega ( $\omega$ ), Fiabilidad compuesta (FC), Variancia media extraida (AVE)

\subsection{Validez}

Se obtuvieron evidencias de validez de convergente y discriminante de las escalas utilizadas. El análisis se realizó de manera jerárquica, considerando los constructos de primer y segundo orden. La validez convergente se evidenció mediante la varianza media extraída (AVE) de cada constructo (Tabla 3). A excepción de la escala de participación, la varianza media extraída de las demás escalas fueron superiores al punto de corte sugerido (.50) Hair, Hult, Ringle, \& Sarstedt (2017).

Asimismo, las cargas factoriales de los indicadores oscilaron entre .720 y .967. Estos valores demuestran la validez convergente de las escalas empleadas. Si bien la AVE de escala de participación fue de .483 y las cargas factoriales de los indicadores oscilaron entre .508 y .799; el constructo se mantuvo en el modelo estructural porque su medición demostró ser fiable y por su 
relevancia conceptual en el modelo explicativo propuesto.

La validez discriminante de todos los constructos del modelo propuesto se examinó aplicando: a) El criterio de Fornell-Larcker y b) El criterio de Heterotrait Monotrait Ratio (HTMT). En ambos casos se corroboró que las dimensiones y variables de primer orden representan constructos únicos conformados por los indicadores que se asignaron teóricamente. Aplicando el primer criterio se confirmó que los constructos comparte más varianza con sus indicadores en comparación a otros constructos Por esta razón, en la Tabla 4 se aprecia que la raíz cuadrada de la varianza media extraída (AVE) compartida entre el constructo y sus indicadores es mayor a las correlaciones con otros constructos Hair, Hult, Ringle, \& Sarstedt (2017).

Tabla 4. Validez discriminante según el criterio de Fornell-Larcker

\begin{tabular}{|c|c|c|c|c|c|c|c|c|}
\hline & CAP & $\cos$ & IE & IPSE & PAR & POS & RHTRC & VIN \\
\hline CAP & 0.894 & & & & & & & \\
\hline $\cos$ & 0.496 & 0.967 & & & & & & \\
\hline IE & 0.533 & 0.409 & 0.832 & & & & & \\
\hline IPSE & 0.465 & 0.297 & 0.398 & 0.803 & & & & \\
\hline PAR & 0.173 & 0.051 & 0.209 & -0.015 & 0.676 & & & \\
\hline POS & 0.605 & 0.525 & 0.444 & 0.574 & 0.130 & 0.806 & & \\
\hline RHTRC & 0.675 & 0.475 & 0.668 & 0.356 & 0.273 & 0.484 & 0.733 & \\
\hline VIN & 0.255 & 0.158 & 0.310 & 0.224 & 0.280 & 0.162 & 0.341 & 0.873 \\
\hline
\end{tabular}

Considerando las limitaciones del criterio de Fornell-Larcker y para determinar con mayor precisión la validez discriminante, también se utilizó el criterio HTMT (Tabla 5). Como se esperaba, los coeficientes HTMT se encuentran por debajo del punto de corte conservador (0.90) y los intervalos de confianza no incluyen a la unidad Hair, Hult, Ringle, \& Sarstedt (2017). Estos hallazgos ratifican el cumplimiento de la validez discriminante de las dimensiones y variables de primer orden incluidas en el modelo estructural propuesto.

Tabla 5.Validez discriminante usando el criterio de Heterotrait Monotrait Ratio (HTMT)

\begin{tabular}{|c|c|c|c|c|c|c|c|}
\hline & CAP & COS & IE & IPSE & PAR & POS & RHTRC \\
\hline \multirow{2}{*}{$\operatorname{COS}$} & 0.593 & & & & & & \\
\hline & {$[0.435 ; 0.726]$} & & & & & & \\
\hline \multirow{2}{*}{ IE } & 0.659053665 & 0.461 & & & & & \\
\hline & {$[0.526 ; 0.788]$} & {$[0.329 ; 0.581]$} & & & & & \\
\hline \multirow{2}{*}{ IPSE } & 0.633 & 0.343 & 0.524 & & & & \\
\hline & {$[0.484 ; 0.787]$} & {$[0.206 ; 0.498]$} & {$[0.387 ; 0.663]$} & & & & \\
\hline \multirow{2}{*}{ PAR } & 0.305 & 0.096 & 0.341 & 0.224 & & & \\
\hline & {$[0.166 ; 0.516]$} & {$[0.051 ; 0.288]$} & {$[0.206 ; 0.530]$} & {$[0.164 ; 0.431]$} & & & \\
\hline
\end{tabular}




\begin{tabular}{|c|c|c|c|c|c|c|c|}
\hline POS & $\begin{array}{c}0.815 \\
{[0.667 ; 0.946]}\end{array}$ & $\begin{array}{c}0.637 \\
{[0.501 ; 0.759]}\end{array}$ & $\begin{array}{c}0.561 \\
{[0.416 ; 0.690]}\end{array}$ & $\begin{array}{c}0.770 \\
{[0.635 ; 0.903]}\end{array}$ & $\begin{array}{c}0.270 \\
{[0.161 ; 0.486]}\end{array}$ & & \\
\hline $\begin{array}{l}\text { RHTR } \\
\text { C }\end{array}$ & $\begin{array}{l}0.861 \\
{[0.772 ; 0.947} \\
]\end{array}$ & $\begin{array}{l}0.540 \\
{[0.421 ; 0.646} \\
]\end{array}$ & $\begin{array}{l}0.794 \\
{[0.714 ; 0.873} \\
]\end{array}$ & $\begin{array}{l}0.484 \\
{[0.341 ; 0.630} \\
]\end{array}$ & $\begin{array}{l}0.469 \\
{[0.309 ; 0.658} \\
]\end{array}$ & $\begin{array}{l}0.624 \\
{[0.503 ; 0.733} \\
]\end{array}$ & \\
\hline VIN & $\begin{array}{c}0.319 \\
{[0.194 ; 0.454]}\end{array}$ & $\begin{array}{c}0.179 \\
{[0.067 ; 0.309]}\end{array}$ & $\begin{array}{c}0.363 \\
{[0.250 ; 0.484]}\end{array}$ & $\begin{array}{c}0.308 \\
{[0.190 ; 0.439]}\end{array}$ & $\begin{array}{c}0.458 \\
{[0.291 ; 0.654]}\end{array}$ & $\begin{array}{c}0.202 \\
{[0.098 ; 0.344]}\end{array}$ & $\begin{array}{c}0.409 \\
{[0.287 ; 0.531} \\
]\end{array}$ \\
\hline
\end{tabular}

Nota: Capacitaciones (CAP); Costos en RRHH (COS); Impacto Económico (IE); Influencia de la política social y económica (IPSE); Participación (PAR); Posicionamiento (POS); Potenciales recursos Humanos para la transferencia de conocimiento (RHTRC); Vinculación empresa-universidad (VIN). Los valores entre corchetes representan el intervalo de confianza del 95\% se utilizó el método Bias-Corrected and Accelerated (Bca), y fueron obtenidas con un bootstrap de 5,000 submuestras.

4.3 Importancia de los factores internos $y$ externos para la vinculación empresauniversidad

El análisis descriptivo mostró que los directivos de las empresas consideran al potencial de los recursos humanos para la transferencia de conocimiento, el impacto económico y la capacitación proveída a los miembros de las organizaciones como las razones más importantes para vincularse con las universidades y el Programa Dual (Figura 1)

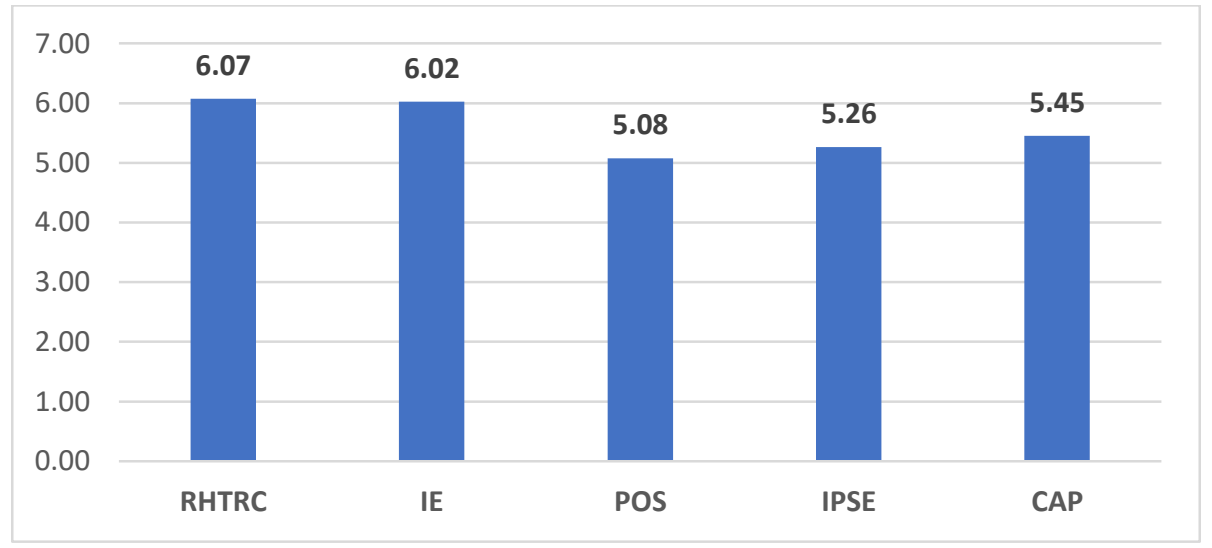

Figura 1. Importancia de los factores para la vinculación empresa-universidad

Nota: Se compararon las medias ponderadas de cada factor. Potenciales recursos Humanos para la transferencia de conocimiento (RHTRC); Impacto Económico (IE); Posicionamiento (POS); Influencia de la política social y económica (IPSE); Participación (PAR); Capacitaciones (CAP).

Considerando los indicadores de cada uno de los factores, los directivos valoraron como muy o extremadamente importante que los estudiantes cuenten con las habilidades que la empresa busca (89.9\%) y se encuentren ávidos para adquirir conocimientos (83.8\%), Además, la oportunidad para desarrollar nuevos proyectos en la empresa $(80.7 \%)$, el ratio de costo-beneficio positivo (80.4\%) y el cumplimiento del compromiso socialmente responsable (74.3) también son evaluados mayoritariamente como muy o extremadamente importantes (Tabla 6). 
Tabla 6.Analisis descriptivo de las dimensiones de los factores internos y externos

\begin{tabular}{|c|c|c|c|c|c|c|}
\hline \multirow[b]{2}{*}{ Escala /Ítems } & \multirow[b]{2}{*}{ Media } & \multirow[b]{2}{*}{ DE } & \multicolumn{4}{|c|}{ Importancia } \\
\hline & & & Nada & $\begin{array}{l}\text { Mínimamente } \\
\text { o poca }\end{array}$ & $\begin{array}{c}\text { Ligera o } \\
\text { moderadamente }\end{array}$ & $\begin{array}{c}\text { Muy o } \\
\text { extremadamente }\end{array}$ \\
\hline $\begin{array}{l}\text { Potencial de los recursos humanos para } \\
\text { la transferencia de conocimiento }\end{array}$ & 30.35 & 3.65 & & & & \\
\hline $\begin{array}{l}\text { La retroalimentación de la universidad } \\
\text { sobre los resultados de las investigaciones } \\
\text { y/o proyectos desarrollados en la empresa }\end{array}$ & 5.70 & 1.28 & 1.0 & 6.1 & 29.4 & 64.5 \\
\hline $\begin{array}{l}\text { Los estudiantes cuentan con habilidades } \\
\text { que la empresa busca }\end{array}$ & 6.32 & 0.82 & 0.0 & 1.0 & 9.1 & 89.9 \\
\hline $\begin{array}{l}\text { Los estudiantes proveen ideas innovadoras } \\
\text { a la empresa }\end{array}$ & 6.12 & 0.96 & 0.3 & 1.0 & 19.6 & 79.1 \\
\hline $\begin{array}{l}\text { La formación de estudiantes en el sistema } \\
\text { dual simplifica la formación de } \\
\text { especialistas y futuros colaboradores }\end{array}$ & 6.17 & 0.94 & 0.3 & 0.7 & 18.9 & 80.1 \\
\hline $\begin{array}{l}\text { Los estudiantes aprendices están ávidos } \\
\text { para adquirir conocimiento }\end{array}$ & 6.24 & 0.83 & & 0.7 & 15.5 & 83.8 \\
\hline Desarrolla investigaciones empresariales & 5.50 & 1.32 & 1.7 & 6.4 & 34.1 & 57.8 \\
\hline Impacto económico & 24.09 & 3.20 & & 0.0 & 0.0 & \\
\hline $\begin{array}{l}\text { Apoya en el cumplimiento de objetivos } \\
\text { estratégicos }\end{array}$ & 5.92 & 1.01 & 0.3 & 2.8 & 24.0 & 72.8 \\
\hline $\begin{array}{l}\text { Mejora el nivel de productividad en la } \\
\text { empresa }\end{array}$ & 5.99 & 0.97 & & 2.4 & 24.3 & 73.3 \\
\hline $\begin{array}{l}\text { La oportunidad para desarrollar nuevos } \\
\text { proyectos en la empresa }\end{array}$ & 6.10 & 0.97 & 0.3 & 1.0 & 17.9 & 80.7 \\
\hline El ratio de costo-beneficio positivo & 6.09 & 0.86 & 0.3 & 0.7 & 18.6 & 80.4 \\
\hline Posicionamiento & 15.24 & 3.70 & & & & \\
\hline Las recomendaciones de otras empresas & 5.28 & 1.53 & 5.4 & 6.1 & 34.5 & 54.1 \\
\hline Su ubicación geográfica es estratégica & 5.05 & 1.61 & 5.1 & 11.8 & 37.2 & 45.9 \\
\hline Emplea un marketing agresivo & 4.90 & 1.45 & 3.7 & 11.1 & 50.0 & 35.1 \\
\hline Capacitación & 10.91 & 2.39 & & & & \\
\hline $\begin{array}{l}\text { Brinda capacitaciones al personal de la } \\
\text { empresa }\end{array}$ & 5.40 & 1.36 & 1.7 & 7.1 & 37.8 & 53.4 \\
\hline $\begin{array}{l}\text { Promueve la formación de instructores } \\
\text { empresariales }\end{array}$ & 5.50 & 1.31 & 1.4 & 6.4 & 35.8 & 56.4 \\
\hline $\begin{array}{l}\text { Influencia de la política social y } \\
\text { económica }\end{array}$ & 15.78 & 3.72 & & & & \\
\hline La obtención de beneficios tributarios & 4.67 & 1.80 & 10.1 & 13.5 & 37.5 & 38.9 \\
\hline $\begin{array}{l}\text { Cumplir con las políticas del estado de } \\
\text { forma constructiva o impositiva }\end{array}$ & 5.16 & 1.63 & 6.1 & 9.1 & 35.5 & 49.3 \\
\hline $\begin{array}{l}\text { Cumplir con el compromiso socialmente } \\
\text { responsable }\end{array}$ & 5.96 & 1.13 & 1.4 & 1.4 & 23.0 & 74.3 \\
\hline
\end{tabular}




\subsection{Impacto de los factores internos,} externos, de costos y participación sobre la Vinculación con la

Universidad

En el modelo conceptual propuesto se formuló que la vinculación debía estar alineada a la satisfacción/compromiso de las empresas participantes en el Programa Dual explicada por factores externos (la ubicación geográfica de las universidades, sus estrategias de marketing y posicionamiento, el cumplimiento de exigencias del estado y expectativas de los grupos de interés, y la conveniencia en términos de beneficios tributarios y capacitación proveída por las universidades) y factores internos (la captación de recursos humanos calificados, las mejoras en innovación, beneficios en el plano económico y de la productividad) que actúan como predictores de una mayor o menor vinculación. Por otro lado, se planteó que los factores internos y externos influyen sobre la vinculación de las empresas indirectamente a través de dos variables mediadoras: la reducción de los costos de capacitación y la participación de las empresas en proyectos académicos promovidos por las universidades. Además, se formuló que estas dos últimas variables impactarían directamente en la vinculación de las empresas con la Universidad a través del Programa Dual.

Los resultados mostraron que las variables incluidas en el modelo explican el $16,2 \%$ de la vinculación de las empresas con la Universidad con Programa Dual $\left(\mathrm{R}^{2}=\right.$ 0.162). Los factores internos (Beta $=0.238, \mathrm{p}$ $=.002)$ y factores externos (Beta $=0.373, \mathrm{p}<$ .000) impactan de forma directa sobre a la disminución de los costos asumidos por las empresas en el ámbito de la selección y capacitación del personal, explicando el $31.3 \%$ de su variación. Sin embargo, la reducción de costos $($ Beta $=-0.020, \mathrm{p}=.770)$ $\mathrm{y}$ los factores externos $($ Beta $=0.104, \mathrm{p}=.221)$ no afectan a la vinculación de las empresas (Figura 2). Por otro lado, los factores internos y externos explican de forma conjunta el $8 \%$ de la participación.

Las variables que predicen de manera significativa y directa una mayor vinculación con Universidades con Programa Dual son los factores internos (Beta $=0.231, \mathrm{p}=.006) \mathrm{y}$ la participación de las empresas en acciones conjuntas con las universidades (Beta = $0.203, \mathrm{p}<.000)$. 


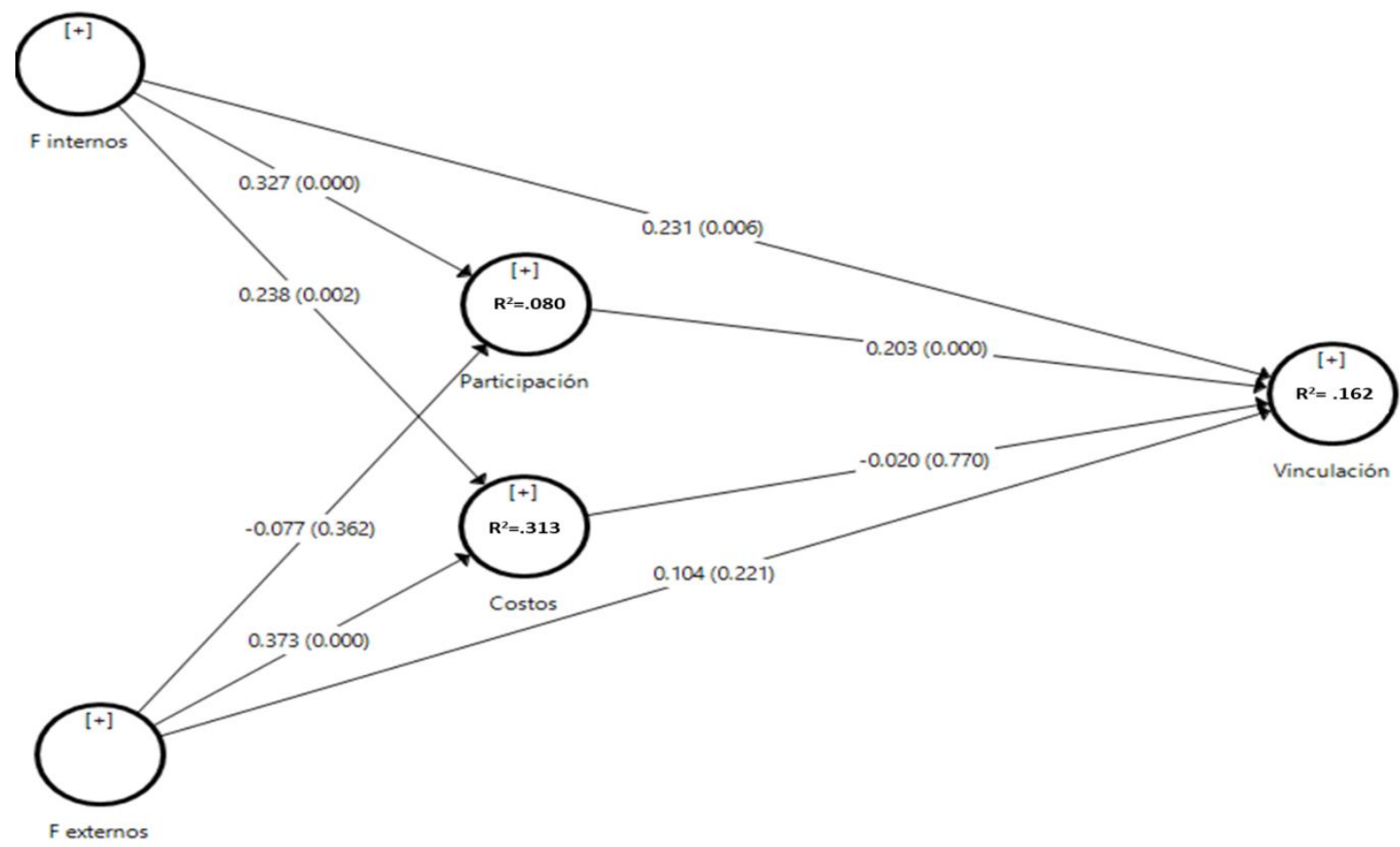

Figura 3. Resultados de Ecuaciones Estructurales y la Relación de los Factores con la Vinculación Ecuaciones estructurales de varianza que explica el impacto de los factores internos y externos sobre la satisfacción-compromiso con el Programa Dual.

Fuente: Encuesta estructurada a 279 conformado por gerentes generales, gerentes de recursos humanos y directivos de empresas.

Los factores internos (la oportunidad de captar talento humano capacitado y con competencias necesarias para la empresa, el acceso a nuevas ideas para la innovación, y las posibilidades para desarrollar acciones que mejoren la gestión empresarial, faciliten el logro de los objetivos estratégicos y eleven la productividad) impactan directamente sobre la participación de las empresas $($ Beta $=$ 0.327, $\mathrm{p}<.000$ ). Estos factores también generan un efecto indirecto sobre la vinculación de las empresas gracias al rol mediador de la participación; es decir, cuando los miembros de las empresas participan en actividades académicas/empresariales y proyectos conjuntos, el impacto sobre la vinculación de las empresas se incrementa.

Es preciso señalar que la participación actúa como una variable mediadora parcial o complementaria al efecto de los factores internos sobre la vinculación (Hair J. , Hult, Ringle, \& Sarstedt (2019). Esta mediación se corrobora al establecer que el efecto directo $(t=2.734 ; p=0.006) \mathrm{e}$ indirecto $(t=2.802 ; p=0.005)$ de los factores internos sobre la vinculación son significativos (Tabla 7).

Tabla 7.Efectos indirectos y función mediadora de los factores reducción de costos y participación de las empresas vinculadas a las universidades con Programa Dual

\begin{tabular}{cccccc}
\hline Muestra & Media de & Desviación \\
original & la muestra & estándar & Estadísticos t & P \\
$(|\mathrm{O} / \mathrm{STDEV}|)$ & Valores \\
& $(\mathrm{O})$ & $(\mathrm{M})$ & $(\mathrm{STDEV})$ & & \\
\hline
\end{tabular}




\begin{tabular}{llllll}
\hline F externos -> Costos -> Vinculación & -0.007 & -0.008 & 0.026 & 0.278 & 0.781 \\
F internos -> Costos -> Vinculación & -0.005 & -0.005 & 0.017 & 0.280 & 0.780 \\
F externos -> Participación -> Vinculación & -0.016 & -0.016 & 0.018 & 0.862 & 0.389 \\
\hline F internos -> Participación -> Vinculación & 0.066 & 0.070 & 0.024 & 2.802 & 0.005 \\
\hline
\end{tabular}

Fuente: Encuesta estructurada a 279 conformado por gerentes generales, gerentes de recursos humanos y directivos de empresas.

Se estableció que las relaciones entre los constructos analizados en el modelo propuesto son estables (Tabla 8). La técnica de Bootstrapping confirmó que los coeficientes beta estandarizados son semejantes a los parámetros poblacionales y estadísticamente significativos $(\mathrm{t}>1.96$, nivel de significancia del $5 \%, \mathrm{y} p<0.05)$.

Tabla 8.Significancia de los coeficientes de trayectoria (beta) entre los factores internos, factores externos, la disminución de costos de selección/capacitación, participación y satisfacción/compromiso

\begin{tabular}{lrrrrr}
\hline $\begin{array}{l}\text { Relación entre dimensiones (valores } \\
\text { Beta) }\end{array}$ & $\begin{array}{c}\text { Muestra } \\
\text { original } \\
(\mathrm{O})\end{array}$ & $\begin{array}{c}\text { Promedio } \\
\text { Muestral } \\
(\mathrm{M})\end{array}$ & $\begin{array}{c}\text { Error } \\
\text { estándar }\end{array}$ & Estadísticos t & $\mathrm{p}$ \\
\hline Costos -> Vinculación & -0.020 & -0.021 & 0.067 & 0.293 & 0.770 \\
F. externos-> Costos en RRHH & 0.373 & 0.382 & 0.077 & 4.853 & 0.000 \\
F. externos-> Participación & -0.077 & -0.077 & 0.084 & 0.911 & 0.362 \\
F. externos -> Vinculación & 0.104 & 0.106 & 0.085 & 1.224 & 0.221 \\
F. internos -> Costos en RRHH & 0.238 & 0.233 & 0.076 & 3.139 & 0.002 \\
F. internos -> Participación & 0.327 & 0.334 & 0.070 & 4.672 & 0.000 \\
F. internos -> Vinculación & 0.231 & 0.233 & 0.085 & 2.734 & 0.006 \\
Participación -> Vinculación & 0.203 & 0.207 & 0.053 & 3.814 & 0.000 \\
\hline Fun
\end{tabular}

Fuente: Técnica de re muestreo (Bootstrapping - 5,000 veces).

Finalmente en la tabla 9 se muestra el resumen de las hipótesis propuestas y los resultados logrados.

Tabla 9. Resultados de las hipótesis planteadas.

\section{Hipótesis propuesta}

H1. Los factores internos son determinantes en la vinculación empresa - universidad

H1a. Los potenciales recursos humanos para transferir conocimiento que provee la universidad son determinantes para la vinculación con la empresa

H1b. El impacto económico generado por el desarrollo de proyectos donde la universidad participa son determinantes para la vinculación con la empresa

H2. Los factores externos son determinantes en la vinculación empresa - universidad

H2a. Las capacitaciones que brindan las universidades son determinantes para la vinculación con la empresa

H2b. La influencia del estado política, social y económica son determinantes para la vinculación con la empresa
Resultado

Aprobado

Aprobado

Aprobado

Rechazado

Rechazado

Rechazado 
H2c. El posicionamiento que tiene la universidad es un determinante para la vinculación con la empresa

H3. La participación de los representantes de la empresas en actividades académicas incrementa la relación entre los factores y la vinculación empresa - universidad

\section{Discusión de resultados}

El estudio se enfocó en identificar los determinantes que vinculan la empresa con la universidad que cuentan con Programa Dual en Latinoamérica. Se tomó como grupo de estudio a las empresas y universidades de la Red DHLA. Por lo que podemos discutir los siguientes resultados:

1) Se aplicó la metodología de Ecuaciones Estructurales con Mínimos Cuadrados Parciales (SEMPLS). El resultado de los análisis del modelo de medida y estructural se realizaron con el paquete estadístico SmartPLS 3.2.3 Ringle, Wende, \& Becker (2017) logrando aplicar una metodología que a través de datos cuantitativos explican las determinantes de la vinculación empresa con la universidad, complementando lo propuesto por DHLA (2018)

2) $\mathrm{Se}$ ha validado el modelo e identificado los factores determinantes de la vinculación empresa universidad aplicado a un programa universitario como se planteaba inicialmente, en este caso fue el Programa Dual que es desarrollado en universidades de Latinoamérica miembros de la Red Duale Hochschule Latinoamérica (DHLA) que tienen relación con empresas donde los estudiantes universitarios tuvieron participación.
3) Los factores internos como determinantes de la vinculación, se puede concluir que las empresas valoran la propuesta de las universidades de proveer recursos humanos para la transferencia de conocimiento, el personal que se incorpora a las empresas provenientes de las universidades con programa dual han demostrado contar con habilidades que las empresas requieren además de su alto espíritu innovador, el recurso humano que se incorpora en la empresa se adecua fácilmente a las actividades laborales y tiene una rápida comprensión de su entorno. Los empresarios han valorado el impacto económico que genera desarrollar proyectos en convenio con la universidad donde se cuenta con practicantes para la ejecución de los mismos. Por consiguiente se puede establecer una coincidencia con la postura de (Perkmann \& Walsh, 2007) que considera que las empresas valoran al personal que le genera valor calificándolo como personal de alta calidad.

4) Los factores externos como determinante de la vinculación, las empresas no consideran importante que la manera de vincularse con la universidad se sostenga por el tipo de capacitaciones recibidas por parte del programa dual, tomando en cuenta que la capacitación obligatoria que se 
desarrolla es la formación de instructores, probablemente las empresas valorarían otros tipos de capacitaciones mejor alineados a sus necesidades. Otro elemento de los factores externos que no han sido considerados determinantes por parte de los empresarios es que a pesar de tener que cumplir con la normativa política, legal de los países que se han estudiado, consideran que no es un incentivo significativo para lograr vincular la empresa con la universidad. El resultado del estudio no es concluyente para rechazar la propuesta de (Larrue, 2000). Que propone que las políticas públicas favorecen las interrelaciones entre los actores económicos.

5) Respecto a la relación de los factores internos y factores externos, con los costos de formación de RRHH y la vinculación, como se ha mencionado las empresas reconocen que contar con estudiantes de universidades del Programa Dual es beneficioso para sus intereses, por ello prefieren contratar a un estudiante que ya viene formado, esto coincide con lo propuesto por Waples (2003). Sin embargo se evidencia que los empresarios frente a la posibilidad de asumir ciertos costos de formación de potenciales recursos humanos el escenario no cambia para algunos y por el contrario el interés de vinculación disminuye. Se debe tener en cuenta que éste análisis es genérico para todas las empresas que participan, indiferentes de su tamaño, es probable que, al momento de realizar un análisis por tamaño de empresa (grandes, medianas y pequeñas), esta relación de vinculación podría variar.

6) Respecto a la relación de los factores internos, con la participación y la vinculación, la participación de los representantes de las empresas en actividades académicas como el intercambio de experiencias empresariales donde los gerentes son expositores y cuando los gerentes son considerados para participar en la evaluación y desarrollo de los planes de estudio para la formación de profesionales genera en las empresas un incentivo para vincularse a las universidades con Programa Dual. Este resultado coincide con lo propuesto por Bloedon \& Stokes (1994), que propone la difusión de conocimiento con la participación activa de empresarios consolidado con un sistema de comunicación que consolide la relación entre los socios y el éxito del vínculo Bonaccorsi \& Piccaluga (1994); Chisholm, (1996); Child \& Faulkner (1998). Inzelt (2004). Kharazmi (2006).

7) Respecto a la relación de los factores externos, con la participación y la vinculación, los factores externos no evidencian algún tipo de influencia en la participación de los empresarios en el entorno académico y por consecuencia no influyen en la vinculación.

\section{Conclusiones.}

1. El Programa Dual en Latinoamérica desarrollado por las universidades miembro de la Red DHLA, viene dando resultados positivos en su vinculación con las empresas

2. Se ha determinado que los estudiantes que han sido formados en 
las universidades con Programa Dual en Latinoamérica son reconocidos como un recurso humano de alta calidad por las empresas y contribuyen en el desarrollo económico de la empresa a través de la ejecución de proyectos que desarrollan junto a la universidad.

3. Es importante que los gerentes y representantes de las empresas participen en la elaboración de los planes de estudio de las universidades y compartan experiencias en foros académicos.

4. Para futuras investigaciones podría ampliar los factores de estudio como la edad de los participantes, el dominio de herramientas tecnológicas, el manejo de idiomas, escala de sueldos que ofrecen las empresas, el plan de estudios y plan de desarrollo profesional.

5. El estudio contribuye con el marco teórico existente. 


\section{Referencias}

Alvarado-Borrego, A. (2000). Vinculación universidad - empresa y su contribución al desarrollo regional. Ra Ximhai, 5(3), 407-414. Recuperado el 2009

Anderson, E. (Mayo de 1998). Customer Satisfaction and Word of Mouth. Journal of Service Research, 5-17.

Antonelli, C. (2000). Collective knowledge communication and innovation: the evidence of technological districts. Regional Studies, 34, 535-547.

Arocena, R., \& Sutz, J. (2005). Latin American Universities: from an Original Revolution to an Uncertain Transition. Higher Education, 50(4), 573-592.

Berstelmann Stiftung. (2013). El sistema dual en Alemania. ¿Es posible transferir el modelo al extranjero? . Fundación Berstelsmann.

Bishop, K., D'Este, P., \& Neely, A. (2011). Gaining from interactions with universities: Multiple methods for nurturing absorptive capacity. Research Policy. 40(1), 30-40. doi:doi:10.1016/j.respol.2010.09.009

Bloedon, R. V., \& Stokes, D. R. (1994). Making University/Industry Collaborative Research Succeed. Research-Technology Management, 37(2), 44-48. doi:10.1080/08956308.1994.11670969

Bloedon, R., \& Stokes, D. (1994). Making University/Industry Collaborative Research Succeed. Research-Technology Management, 37(2), 44-48. doi:10.1080/08956308.1994.11670969

Bock, M. (2018). Modelo Dual Alemán. Una base importante para el éxito económico. En DHLA, Modelo Dual de Educación con sabor Latino (págs. 11-16). Bogota: Ediciones Carrera.

Bonaccorsi, A., \& Piccaluga, A. (1994). A theoretical framework for the evaluation of universityindustry relationships, R. R\&D Management, 24 (3), 229-247.

Bonaccorsi, A., \& Piccaluga, A. (1994). A theoretical framework for the evaluation of universityindustry relationships. 24(3), 229-247. doi:10.1111/j.1467-9310.1994.tb00876.x

Botello, H. (2014). Condiciones y determinantes de la internacionalización de las empresas industrializadas latinoamericanas. Centro de investigación de la Universidad Pacífico, XLI(75), 47-78.

Botero, J., Álvarez, F., \& Gonzales, M. (2012). Modelo de internacionalización para pymes Colombianas. AD. minister. Universidad EAFIT, 20, 63-90.

Bozeman, B., Fay, D., \& Slade, C. P. (2013). Research Collaboration in Universities and Academic Entrepreneurship: the-State-of-theArt', Journal of Technology Transfer. 38(1), 1-67.

Cardozo, P., Chavarro, A., \& Ramírez, C. (2004). Teorias de internacionalización. Global business today.

Child, J., \& Faulkner, D. (1998). Strategies of Cooperation: Managing Alliances, Networks, and Joint Ventures.

Chisholm, R. (1996). On the meaning of networks. 21(2), 216-235.

Cohen et al. (2002). Links and impacts: the influence of public research on industrial R\&D. Management Science, 48(1), 1-23.

Colciencias. (09 de Agosto de 2018). Departamento Administrativo de Ciencia, Tecnología e Innovación. Obtenido de Colciencias web site: http://www.colciencias.gov.co/portafolio/innovacion/transferencia-conocimiento

Coleman, J. S. (01 de January de 2003). Social capital in the creation of human capital. Foundations of Social Capital. 159-184.

Cooke, P. (2001). Regional innovation systems, clusters and the knowledge economy. Industrial and Corporate Change 10 . 10(4), 945-974.

D'Este, P., García Quevedo, J., \& Mas-Verdú, F. (mayo de 2014). Transferencia del Conocimiento del Modelo Transaccional al Relacional. Mediterráneo Económico, 25, 279- 296. 
Recuperado el 10 de 02 de 2019, de www.publicacionescajamar.es/publicacionesperiodicas/mediterraneo-economico/

DHLA, D. H. (2018). Modelo Dual de Educación con Sabor Latino. Bogota: Ediciones Carrera 7 SAS. doi:978-958-99643-2-3

Dill, D. D. (01 de June de 1995). University-industry entrepreneurship: The organization and management of American university technology transfer units. Higher Education, 29(4), 369-384.

Doyle, P. (1989). Building successful brands. Journal of Marketing Management, 75-95.

Dualvet - Programa de Aprendizaje Permanente (Comisión Europea). (2015). Cómo implementar con éxito la formación profesional dual. Zaragoza: Selenus. Recuperado el 10 de febero de 2019, de www.dualvet.eu/docs/productos/Dualvet_ES.pdf

Etzkowitz , H., \& Leydesdorff, L. (2000). The dynamics of innovation: from national systems and "Mode 2 " to a triple helix of university-industry-government relations. Research Policy, 29(2), 109-125.

Fernández et al. (2000). Las relaciones universidad-empresa: entre la transferencia de resultados y el aprendizaje regional. . Asociación para el Desarrollo de la Ciencia y la Tecnología.

Fernández, M., Peña, I., \& Hernández, F. (2008). Factores determinantes del éxito exportador. El papel de la estrategia expotadora en las cooperativas agrarias. Revista de Economía Pública, Social y Cooperativa, 39 - 64.

García-Aracil, A., \& Fernández, D. (01 de March de 2008). Industry-University Interactions in a Peripheral European Region: An Empirical Study of Valencian Firms. Regional Studies,, 42(2), 215-227.

Gupta, A. k., \& Singhal, A. (1993). Managing Human Resources for Innovation and Creativity. Research-Technology Management, 36 41-48. doi:doi:10.1080/08956308.1993.11670902

Hair, Hult, Ringle, \& Sarstedt. (2017). A Primer on Partial Least Squares Structural Equation Modeling 2nd Ed. Thousand Oaks: Sage.

Hair, J., Hult, G., Ringle, C., \& Sarstedt, M. (2019). Hair, J., Hult, G., RingleManual de Partial Least Squares Structural Equation Modeling (PLS-SEM). Los Angeles: Omnia Science.

Hair, Ringle, Hult, \& Sarstedt. (2014). A Primer on Partial Least Squares Structural Equation Modeling. Thousand Oaks: Sage.

Hanel, P., \& St-Pierre, M. (01 de July de 2006). Industry-University Collaboration by Canadian Manufacturing Firms*. The Journal of Technology Transfer, 31(4), 485-499.

Howells, J., Ramlogan, R., \& Cheng, S. L. (2012). 'Innovation and university collaboration: paradox and complexity within the knowledge economy' Cambridge Journal of Economics. 36(3), 703-721.

Inzelt, A. (2004). The evolution of university-industry-government relationships during transition. 6-7, 975-995. doi:10.1016/j.respol.2004.03.002

Irigoyen, J., Jiménez, M., \& Acuña, K. (2011). Competencias y educación superior. Revista mexicana de Investigación Educativa. 16(48), 243 - 266.

Jiménez, I. (2007). Determinantes para la internacionalización de pymes mexicanas. Análisis económico, 22(49), 111-131. doi:ISSN: 0185-3937

Keller, K. (2008). Administración Estratégica de marca.

Keller, K. L. (2008). Administración Estratégica de marca. Pearson.

Kenney, M. (1986). Biotechnology: The University-Industrial Complex. New Haven, CT: Yale University Press.

Kharazmi, O. (2006). Transfer of Technology from Universities to Industry. 
Klofsten, M., \& Jones-Evans, ,. (01 de June de 2000). Comparing Academic Entrepreneurship in Europe: The Case of Sweden and Ireland. Small Business Economics,, 14(4), 299-309.

Kornhauser, A. (01 de January de 2001). Knowledge as capital. Biotechnological Intellectual Property at Universities. 5-9.

Kotler, P. (2008). Fundamentos de Marketing .

Kupffer, J. (2005). “Formación Dual en Latinoamérica” . Cámara de Comercio e Industria Boliviano - Alemana.

Lahera, E. (2004). Política y Políticas Públicas. Santiago: CEPAL.

Landry, R., \& Amara, N. (1998). The impact of transaction costs on the institutional structuration of collaborative academic research. Research Policy, 27(9), 901-913. doi:10.1016/s00487333(98)00098-5

Larrue, C. (2000). Analyser les politiques publiques d'environnement. L'Harmattan.

Laursen, K., \& Salter, A. (2004). Searching high and low:what types of firms use universities as a source of innovation? 1201-1215.

Link, A. N., \& Siegel, D. S. (2007). Innovation, entrepreneurship, and technological change. Oxford: Oxford University Press.

López, Martínez, R. E., Medellín, E., Scanlon, A. P., \& Solleiro, J. L. ( 01 de January de 1994). Motivations and obstacles to university industry cooperation (UIC): a Mexican case. 24(1), 17-30. doi:https://doi.org/10.1111/j.1467-9310.1994.tb00844.x

Mansfield, E. (1995). Academic research underlying industrial innovations: sources, characteristics, and financing. Review of Economics and Statistics, 77, 55-65.

Marques et al. (June de 2003). The Macro and micro context of business incubators: a preliminary approach to incubation processes. In: XX IASP World Conference On Science And Technology Parks. Habitats of Excellence-Managing and Promoting Innovation, 113-121.

Marzo, M., Pedraja, M., \& Rivera, P. (2008). Un modelo de relaciones empresa-universidad. Academia Europea de Dirección y Economía de la Empresa.

Mead et al. (1999). Industry/university collaborations: different perspectives heighten mutual opportunities. Journal of Systems and Software, 49(2-3), 155-162. doi:10.1016/s01641212(99)00091-6

Mead. (1999). Different perspectives heighten mutual opportunities. Journal of Systems and Software, 49(2-3), 155-162. doi:10.1016/s0164-1212(99)00091-6

Nunally, J., \& Bernstein, I. (1994). Psychometric theory. New Yorrk: McGraw-Hill.

OIT. (2018). Pespectivas sociales y el empleo en el mundo: Tendencias 2018. Ginebra: Organización Mundial del Trabajo.

OMPI. (16 de Agosto de 2018). Organización Mundial de la Propiedad Intelectual. Obtenido de Organización Mundial de la Propiedad Intelectual sitio web: http://www.wipo.int/aboutip/es/universities_research/ip_knowledgetransfer/faqs/

Parra, R. R. (03 de septiembre de 2002). El Tiempo Casa Editorial. Obtenido de El Tiempo sitio web: https://www.eltiempo.com/archivo/documento/MAM-1366639

Peña, S. (2005). Modelo de selección de personal en base a perfil de competencias. 2(1), 121-144.

Perkmann, M., \& Walsh, K. (2007). University-industry relationships and open innovation: Towards a research agenda. International Journal of Management Reviews. 9(4), 259-280.

Perkmann, M., Valentina, T., Mckelvery, M., Autio, E., Broström, A., D'Este, P., . . . Sobrero, M. (marzo de 2013). Academic engagement and commercialisation: A review of the literature on university-industry relations. Research Policy, 42(2), 423-442. doi:10.1016/j.respol.2012.09.007

Philip Kotler, G. A. (2008). Fundamentos de Marketing. Pearson . 
Reddy, P. (2011). he Evolving Role of Universities in Economic Development: The Case of University-Industry Linkages. 25-49.

Rego, L., Barreira, E., \& Rial, A. (2015). Formación profesional dual. Una comparativa entre el sistema alemán y el incipiente sitema Español. Revista Española de Educación Comparada, $149-166$.

Ringle, C., Wende, S., \& Becker, J. (15 de March de 2017). SmartPLS 3. Boenningstedt: SmartPLS $\mathrm{GmbH}$. Obtenido de www.smartpls.com.

Sabato, J., \& Botana, ,. (Noviembre de 1968). "La ciencia y la tecnología en el desarrollo futuro de América Latina”. Revista de Integración, № 3.

Schartinger et al. (March de 2002). Knowledge interactions between universities and industry in Austria: sectoral patterns and determinants. Research Policy, 31(3), 303-328.

Segarra-Blasco , A., \& Arauzo-Carod, J. (01 de January de 2008). Sources of innovation and industry-university interaction: Evidence from Spanish firms. Research Policy, 37(8), $1283-$ 1295.

Simanaviciene, Z., Zuzeviciute, V., \& Praneviciene, B. (2017). Cooperation between university and business in Lithuania: examples of good practices, University- enterprises cooperation in Asia: trends and challenges. 24-29.

Siqueira et al. (01 de June de 2009). University-industry interactions in an immature system of innovation: Evidence from Minas Gerais, Brazil. Science and Public Policy, 36(5), 373-386. Obtenido de https://doi.org/10.3152/030234209X442016

Slaughter, \& Rhoades. (2004). Academic Capitalism and the New Economy: Markets, State, and Higher Education. Johns Hopkins University Press.

Slaughter, S., \& Leslie, L. (1997). Academic Capitalism: Politics, Policies and the Entrepreneurial University. Johns Hopkins University Press.

Slaughter, S., \& Leslie, L. (1997). Academic Capitalism: Politics, Policies and the Entrepreneurial University. Johns Hopkins University Press.

Solis, J. (2008). Responsabilidad social empresarial, un enfoque alternativo. Análisis económico, 227-252.

Tabares, S. (2012 ). Internacionalización de la pyme Latinoamericana. Referente para el éxito empresarial de Colombia. Ciencias Estratégicas, 119 -132. .

Temple, P. (2006). Branding higher education: illusion or reality. Perspective, 15-19.

Temple, P. (2006). Branding higher education: illusion or reality. 15-19.

Thoenig, J. (1985). Traité de Science Politique. Les Politiques publiques, 4.

Vega, \& Jurado et al. (2008). University-industry relations in Bolivia: implications for university transformations in Latin America. 56(2), 205-220. doi:https://doi.org/10.1007/s10734007-9098-9

Wæraas, A. a. (2009). 'Defining the essence of a university: Lessons from higher education branding'. Higher education, 5, 449-462.

Waples, L. (2003). University-industry partnerships in biomedical engineering. Engineering in Medicine and Biology Magazine, 4, 118-121.

Waples, L. M., \& Ropella, K. M. (2003). University-industry partnerships in biomedical engineering. IEEE Engineering in Medicine and Biology Magazine. 22(4), 118-121. doi:doi:10.1109/memb.2003.1237512. 


\section{Agradecimientos}

Agradecer al Comité Superior Central de la Red Duale Hochschule Latinoamérica (DHLA) y al gerente del proyecto de la DHLA Peter Hirsch por promover espacios de investigación, a los Rectores y Decanos de las Universidades miembro de la Red DHLA por su colaboración y gestión incorporando a sus investigadores al estudio. Un especial agradecimiento a los investigadores, López-Odar, Dennis y Vara-Horna, Arístides, de la Universidad de San Martín de Porres, Perú, a Garcera-Murillo, Mario de la Fundación Universitaria Empresarial de la Cámara de Comercio de Bogotá, Colombia, a Gutiérrez-Posada, Nolberto de la Corporación Universitaria Empresarial Alexander von Humboldt, Colombia, a Orellana-Osorio, Iván de la Universidad de Cuenca, Ecuador, a Cajigas-Romero, Margot y Garcés, Jorge de la Universidad Autónoma de Occidente de Cali, Colombia, a Reséndiz, Ana de la Universidad Interamericana para el desarrollo, México, cada uno de ellos contribuyendo con sus revisiones y complementando información relevante al estudio. También extender nuestro agradecimiento al equipo operativo de cada institución para el desarrollo de trabajo de campo entre ellos a Juan Patrón, Hilda Ruíz, Karla Montoya, Geider Solís, Landy Estrada, María Aguilar, Francisco Rueda, Luis de la Cruz, Ramón Abán, Martín Flores, Juan Rodríguez, Diana Rubiano, Claudia Cardona, Pedro Mora, Juan Andrade, Marcia Pesántez, Adrián Alvarado, Melissa More.

Es una investigación que se caracteriza por el trabajo en equipo de representantes de instituciones comprometidos con el desarrollo de la región. 